\title{
Functional analysis and exterior calculus on mixed-dimensional geometries
}

\author{
Wietse M. Boon ${ }^{1}$ (D) . Jan M. Nordbotten ${ }^{2}$ (D) . Jon E. Vatne ${ }^{3}$ (D)
}

Received: 29 October 2019 / Accepted: 12 June 2020 / Published online: 12 July 2020

(c) The Author(s) 2020

\begin{abstract}
We are interested in differential forms on mixed-dimensional geometries, in the sense of a domain containing sets of $d$-dimensional manifolds, structured hierarchically so that each $d$-dimensional manifold is contained in the boundary of one or more $d+1$-dimensional manifolds. On any given $d$-dimensional manifold, we then consider differential operators tangent to the manifold as well as discrete differential operators (jumps) normal to the manifold. The combined action of these operators leads to the notion of a semi-discrete differential operator coupling manifolds of different dimensions. We refer to the resulting systems of equations as mixed-dimensional, which have become a popular modeling technique for physical applications including fractured and composite materials. We establish analytical tools in the mixed-dimensional setting, including suitable inner products, differential and codifferential operators, Poincaré lemma, and Poincaré-Friedrichs inequality. The manuscript is concluded by defining the mixed-dimensional minimization problem corresponding to the Hodge Laplacian, and we show that this minimization problem is well-posed.
\end{abstract}

Keywords Mixed-dimensional differential operators · Mixed-dimensional geometry · Exterior calculus

Mathematics Subject Classification $46 \mathrm{C} 05 \cdot 46 \mathrm{E} 35 \cdot 46 \mathrm{~F} 10 \cdot 58 \mathrm{~A} 12 \cdot 58 \mathrm{C} 05 \cdot 58 \mathrm{~J} 10$

\section{Introduction}

Partial differential equations of reduced dimension are common in mathematical modeling, and examples include shells, membranes, fractures, geological formations, etc. (see, e.g., $[2,6,15,28])$. When such structures are embedded into a higher-dimensional surrounding media, it is often advantageous to consider the resulting problem as a mixed-dimensional

Wietse M. Boon

wietse@kth.se

1 Department of Mathematics, KTH Royal Institute of Technology, Stockholm, Sweden

2 Department of Mathematics, University of Bergen, Bergen, Norway

3 Department of Computer Science, Electrical Engineering and Mathematical Sciences, Western Norway University of Applied Sciences, Bergen, Norway 
problem (also referred to as hybrid-dimensional by some authors). To our knowledge, such problems have until present been considered in a case-by-case basis, based on the needs of the various applications. In this paper, we treat the case of hierarchical co-dimension one, that is to say, for an $n$-dimensional domain, we consider manifolds of dimension $n-1$, and their intersections, again manifolds, of dimension $n-2, n-3$, and so on. We refer to this geometric construction as mixed-dimensional.

In order to provide a unified theoretical framework for mixed-dimensional partial differential equations, we use the setting of exterior calculus, identify a suitable notion of spaces of alternating $k$-forms for the mixed-dimensional geometry, and equip the spaces with inner products and norms. We define a discrete-continuous differential operator acting on alternating $k$-forms, and its adjoint, a codifferential operator. We show that our spaces and differential operator form a de Rham complex, with the same cohomology spaces as the full domain.

In the application section, we show that quadratic minimization problems defined in terms of our mixed-dimensional differential operator are well posed. It follows that we can write variational forms of partial differential equations, formally consistent with the strong form of the partial differential equations. Additionally, we show how our findings directly lead to discretization methods using finite element exterior calculus [3] and we present an explicit example of a mixed-dimensional partial differential equation related to flow in fractured porous media.

We note several relationships between our current work and previous literature. Firstly, in our construction, lower-dimensional manifolds are restricted such that they coincide with boundaries of manifolds of one higher (topological) dimension, and their boundaries coincide with manifolds of one lower dimension. As such, the connections between the manifolds are similar to those appearing in Čech complexes, and we arrive at double complexes that have much in common to the Čech-de Rham complexes [10, 34]. However, the details of our construction differ, as we are considering not overlaps of open sets, but actual lower-dimensional manifolds. There is therefore also a parallel to singular cohomology as presented in [10]. Another connection can be found to distributional forms, and in particular the refinement of those developed by Licht [21]. Our work can be seen as generalizing that work from finite-dimensional spaces on simplicial complexes (motivated by polynomial forms and finite element discretizations), to infinite-dimensional spaces (continuous functions and Sobolev spaces) on more general manifolds, not necessarily simplicial. This generalization is essential for our goal of constructing mixed-dimensional partial differential equations corresponding to those found in the cited applications. On the other hand, our constructions are more concrete than the abstract setting of distributional differential forms presented by Melrose [24], where there is no restriction on the locus where a distributional form fails to be represented by ordinary differential forms. This allows us to not only consider Sobolev spaces in the sense of distributional forms, but also explicitly represent these spaces in terms of classical Sobolev spaces on the separate manifolds. The analysis conducted herein provides the theoretical foundation for the research program outlined by the authors for modeling, analysis and numerical discretization of mixed-dimensional physical problems [27].

The key novel contributions of this work are as follows:

1. The use of directed acyclic graphs (DAGs) to represent connections between manifolds comprising a mixed-dimensional geometry (Definition 2.2). 
2. Explicit representation of Sobolev spaces for mixed-dimensional geometries in terms of classical Sobolev spaces on individual manifolds (Lemma 3.10).

3. A cohomology theory extending discrete distributional forms to distributional differential forms which are locally continuous with respect to mixed-dimensional geometries (Theorem 3.3) and the extension of classical results from functional analysis to mixeddimensional function spaces (e.g., Theorems 3.4, 3.5).

4. An explicit link between our mixed-dimensional construction (and thus implicitly also Čech-de Rham complexes and discrete distributional forms) and current research within analysis of physical models for mixed-dimensional partial differential equations in applications (Sect. 4).

\section{Background and notation}

Herein we summarize the geometric setting of interest, and the notational conventions used.

\subsection{Geometric setting}

The precise description of the geometric setting is quite technical, but is required to make the calculations later in the manuscript precise. An intuitive understanding of the geometry can be obtained by considering the examples and figures.

We consider an open domain $Y \subset \mathbb{R}^{n}$, together with its boundary $\partial Y$, which is decomposed into two parts $\partial_{N} Y$ and $\partial_{D} Y$ such that $\partial Y=\partial_{N} Y \cup \partial_{D} Y$ and $\partial_{N} Y \cap \partial_{D} Y=\emptyset$. We are interested in partitions into disjoint, connected manifolds $\Omega_{i}^{d} \subset Y_{N}$, of the domain $Y_{N}=Y \cup \partial_{N} Y$. The partition is indexed by $i \in I$ with $d_{i}$ the dimension, and we denote the set of indices for which $d_{i}=d$ as $I^{d}$. We require that the manifolds $\Omega_{i}^{d_{i}}$ are diffeomorphic to bounded open sets $X_{i} \subset \mathbb{R}^{d_{i}}$. Furthermore, we require that $\bar{X}_{i}$ is a $d_{i}$-manifold with boundary $\bar{X}_{i} \backslash X_{i}$. In the special case that $X_{i}$ is the $d_{i}$-dimensional unit ball, we write $X_{i}=B^{d_{i}}$. We require that $\cup_{i \in I} \Omega_{i}^{d_{i}}=Y_{N}$ disjointly, i.e., that every point in $Y_{N}$ can be associated with a unique $\Omega_{i}^{d_{i}}$. We frequently omit the superscript $d_{i}$, i.e., write $\Omega_{i}=\Omega_{i}^{d_{i}}$, and refer to the collection of manifolds simply as $\Omega$.

In order to precisely discuss this geometry and the restrictions we require, we will represent it using rooted directed acyclic graphs (which we will refer to as DAGs) with coordinate maps. Thus for every domain $\Omega_{i}$ with index $i \in I$, let $\mathfrak{S}_{i}$ be the DAG associated with $i$ of maximal depth $d_{i}$. We will choose a global enumeration of nodes such that the root of DAG $\mathfrak{S}_{i}$ is node $i$. Within each DAG, we denote the descendants of any node $j$ as $I_{j}$. Moreover, we define the subset $I_{i}^{d} \subset I_{i}$ as all descendants $l \in I_{i}$ with associated dimension $d_{l}=d$.

In a slight abuse of terminology, we will refer to the family of all the DAGs as the forest $\mathfrak{F}$, defined as

$$
\mathfrak{F}=\bigcup_{i \in I} \mathfrak{S}_{i}
$$

While we will refer to $\mathfrak{F}$ as a forest, we note that it can be represented by a star graph with each leaf corresponding to the root of DAG $\mathfrak{S}_{i}$ with $i \in I$. When using the interpretation of the forest as a star graph, we will use the index 0 for the (global) root. In order to simplify summations, we slightly abuse notation by using DAGs and the forest also as index sets, e.g., write $j \in \mathfrak{F}$ to denote the indices of all nodes in the forest $\mathfrak{F}$. 
We require that each DAG $\mathfrak{S}_{i}$ is endowed with a family of manifolds and maps, such that for every node $j \in I_{i}$, there exists an orientable manifold $X_{j}$ and a smooth bijective coordinate map $\phi_{i, j}: \bar{X}_{j} \rightarrow \bar{\partial}_{j} X_{i}$, where $\partial_{j} X_{i} \subset \partial X_{i}$ is the image of $\phi_{i, j}\left(X_{j}\right)$. For the root of the DAG $\mathfrak{S}_{i}$, the unique parent is the global root 0 , for which we only require that the mapping is surjective, and use the convention that $\partial_{i} X_{0}=\Omega_{i}$, and thus $\phi_{0, i}: \bar{X}_{i} \rightarrow \bar{\Omega}_{i}$. We require that all maps $\phi_{i, j}$ are diffeomorphisms on $X_{j}$.

Definition 2.1 A rooted DAG $\mathfrak{S}_{i}$ with $i \in I$, is conforming to $\Omega_{i}$ if for all nodes $j \in \mathfrak{S}_{i}$ :

1. There exists a root $s_{j} \in I$ such that $\phi_{0, j}\left(X_{j}\right)=\Omega_{s_{j}}$. Moreover, $s_{i}=i$ for the root $i$.

2. Compound maps telescope in the sense that for every $l \in I_{i}$ with $j \in I_{l}$, it holds that $\phi_{i, j}=\phi_{i, l} \circ \phi_{l, j}$.

3. The descendants uniquely cover the parent node in the sense that $\bigcup_{j \in \mathfrak{S}_{i}} \phi_{i, j}\left(X_{j}\right)=\bar{X}_{i} \backslash \phi_{0, i}^{-1}\left(\partial \Omega_{i} \cap \partial_{D} Y\right)$.

In other words, each point $x_{i}$ in reference domain $X_{i}$ and on its boundary is uniquely associated to a node $j \in \mathfrak{S}_{i}$ and a point $x_{j} \in X_{j}$ such that $x_{i}=\phi_{i, j}\left(x_{j}\right)$. For $x_{i}$ on the boundary $\partial X_{i}$, we have $j \in I_{i}$ a descendant of $i$, whereas for $x_{i}$ in the interior of $X_{i}$, we have $j=i$. All points that are mapped to the physical boundary $\partial_{D} Y$ by $\phi_{0, i}$ are exempt from this rule.

Note that we do not require that $s_{j_{1}} \neq s_{j_{2}}$ for $j_{1}, j_{2} \in I_{i}$, such that $\phi_{0, i}$ may map several sections of the boundary $\partial X_{i}$ to the same $\Omega_{j}$. This allows for the manifolds $X_{i}$ to be mapped to manifolds with various kinds of "loose ends" or "slits", as seen in the examples below. Moreover, it is a consequence of the definition of a conforming DAG that for any $i \in I$ and $j \in I_{i}$, it holds that $I_{j} \subset I_{i}$.

Definition 2.2 A forest $\mathfrak{F}$ is conforming to $\Omega$ if the DAGs $\mathfrak{S}_{i}$ are conforming to $\Omega_{i}$ for all $i \in I$ in the sense of Definition 2.1. The forest is open if $\partial_{D} Y=\partial Y$, and is closed if $\partial_{N} Y=\partial Y$.

Open forests allow for boundary conditions on $\partial Y$ to be applied to the manifolds $\Omega_{i}$ which extend to the boundary. Conversely, closed forests allow for differential equations to be defined on the boundary. Here, and in the following, we will only consider partitions $\Omega$ for which there exists a conforming forest.

Example 2.1 A domain partitioned by a simplicial complex has a conforming forest.

Example 2.2 An allowable 2D partitioning which is not a simplicial complex is given in Fig. 1. Also in the figure is the map of the largest top-dimensional domain, as well as the full forest $\mathfrak{F}$. We note that since $X_{4}$ is not isomorphic to a ball, this partition is also not a cellular complex.

Example 2.3 Figure 2 shows a three-dimensional partition in which two of the manifolds $\left(\Omega_{9}^{3}\right.$ and $\Omega_{8}^{2}$ ) are not isomorphic to a ball. As shown in the right of the figure, the embedded network corresponds to a three-dimensional hole in reference space $X_{9}$. 

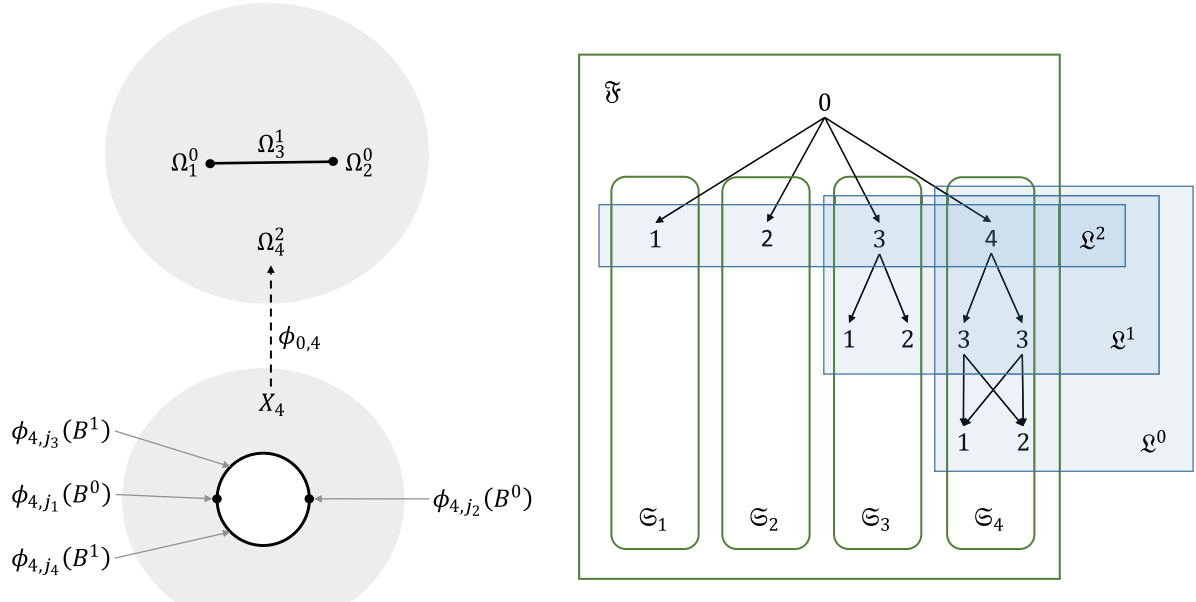

Fig. 1 Example geometry on an open domain in 2D (upper left); the structure of the reference domain $X_{4}$ and the map to $\Omega_{4}^{2}$ (lower left); and the corresponding forest $\mathfrak{F}$ (right). In this example, there are four manifolds (and DAGs), numbered such that $I^{0}=\{1,2\}, I^{1}=\{3\}$ and $I^{2}=\{4\}$. The nodal labels in the DAGs correspond to the identifications of domains $s_{j}$ - the global numbering of nodes is not shown. Note in particular that due to the "slit", $\Omega_{3}^{1}$ corresponds to two parts of the boundary of $\Omega_{4}^{2}$, denoted by $j_{3}$ and $j_{4}$ in the reference domain. Thus, both $\phi_{0, j_{3}}\left(X_{j_{3}}\right)=\Omega_{3}^{1}$ and $\phi_{0, j_{4}}\left(X_{j_{4}}\right)=\Omega_{3}^{1}$ and this is reflected in the DAG $\mathfrak{S}_{4}$ by the double occurrence of the label 3. We have also highlighted groups of nodes whereon function spaces will be defined in later sections
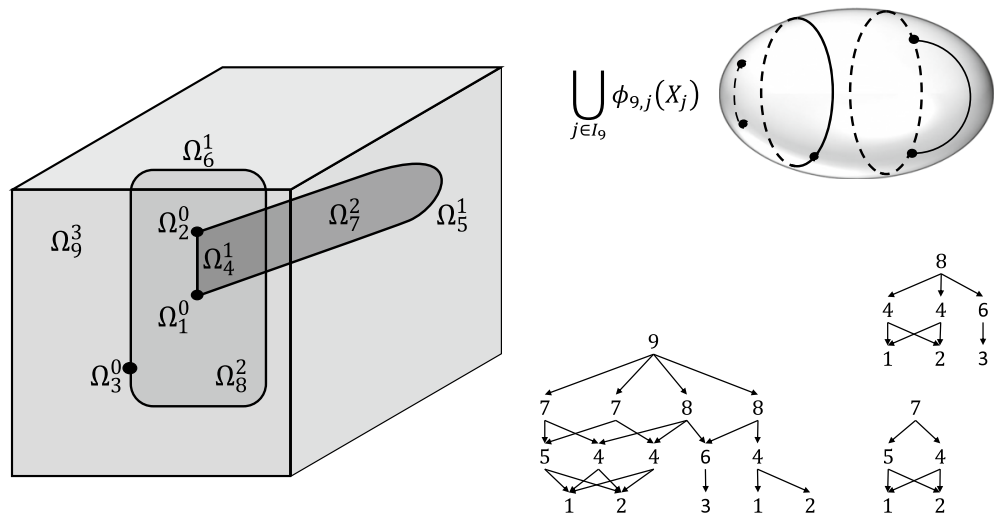

Fig. 2 Example of a geometry which is not a cellular complex in 3D (left). The inner boundary of $X_{9}^{3}$ (top right), and the conforming DAGs with roots in $I^{2}=\{7,8\}$ and $I^{3}=\{9\}$ (bottom right)

Remark 2.1 The previous example illustrates a limitation of our construction, as we have required the manifolds $\Omega_{i}^{d_{i}}$ to be diffeomorphic to bounded open sets in $\mathbb{R}^{d_{i}}$. Thus, with reference to Fig. 2, the 1-manifold $\Omega_{6}^{1}$ cannot be a closed curve, and the 0 -manifold $\Omega_{3}^{0}$ is introduced. We emphasize that this limitation is for notational convenience, in order to be able to refer to a unique coordinate map for each manifold, and that our results extend to manifolds with local coordinate maps. 
Example 2.4 A self-intersecting 2D manifold in 3D is illustrated in Fig. 3. In this example, the top-dimensional domain is not contractible and corresponds to the outside of a torus. The boundary of the top-dimensional domain (logically a torus) is shown together with its decomposition.

A primary object of our study will be function spaces and differential operators with respect to the domain partition $\Omega$ and the corresponding forest $\mathfrak{F}$.

As above, we use a partial to indicate boundaries, such as, e.g., $\partial \Omega_{i}$. The boundary operator on manifolds $\Omega_{i}$ is consistently understood in terms of the reference domains $X_{i}$, such that the notation $\partial_{j} \Omega_{i}$ refers to $\phi_{0, i}\left(\partial X_{i} \cap \phi_{i, j}\left(X_{j}\right)\right)=\phi_{0, j} X_{j}$. Decomposition of boundaries follows the decomposition of the full domain, $\partial \Omega_{i}=\partial_{i} Y \bigcup_{j \in I_{i}} \partial_{j} \Omega_{i}$. Here $\partial_{i} Y=\partial_{D} Y \cap \partial \Omega_{s_{i}}$ and $\partial_{j} \Omega_{i}$ is assumed to have the same coordinate map, up to orientation, as $\Omega_{s_{j}}$. We denote the relative orientation of two such manifolds by $\varepsilon\left(\partial_{j} \Omega_{i}, \Omega_{s_{j}}\right)$ defined as

$$
\varepsilon\left(\partial_{j} \Omega_{i}, \Omega_{s_{j}}\right)= \begin{cases}1, & \text { if the orientations of } \partial_{j} \Omega_{i} \text { and } \Omega_{s_{j}} \text { coincide }, \\ -1, & \text { otherwise. }\end{cases}
$$

We make a final comment on notation. For each $j \in \mathfrak{F}$, we use a hat symbol to denote the corresponding root $\hat{j} \in I$ such that $j \in I_{\hat{j}}$.

\subsection{Fixed-dimensional exterior calculus}

In order to fix notation, we briefly recall some results from exterior calculus. We refer the reader to more comprehensive texts for a complete introduction (some instructive and relevant works are $[1,3,10,18,22,32]$, noting that in general throughout the text we will mostly be consistent with the notation as used in [3] with the exception that for a manifold $\Omega_{i}$, we denote the spaces of alternating $k$-forms

$$
\Lambda^{k}\left(\Omega_{i}\right) \quad \text { for } \quad k \in\left\{0,1, \ldots, d_{i}\right\} .
$$

We will frequently omit the dependence on the domain when no confusion may arise. Spaces of alternating $k$-forms are connected by the wedge product, so that for $a \in \Lambda^{k_{1}}$ and $b \in \Lambda^{k_{2}}$, the product $a \wedge b \in \Lambda^{k_{1}+k_{2}}$. The wedge product has the property that $a \wedge b=(-1)^{k_{1} k_{2}} b \wedge a$.

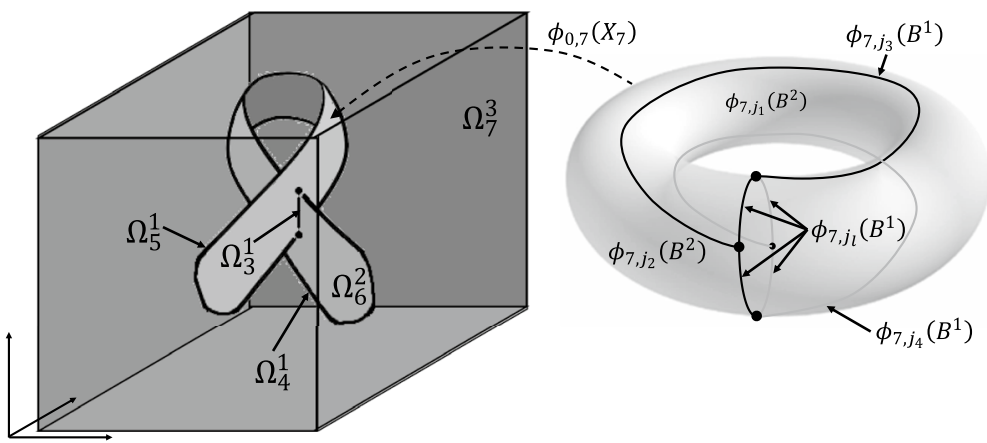

Fig. 3 Example of a permissible geometry in 3D (left), the partitioned inner boundary $\bigcup_{j \in I_{7}} \phi_{7, j}\left(B^{d_{j}}\right) \subset \partial X_{7}^{3}$ of the pre-image of the top-dimensional domain (right) 
We define the space of alternating $k$-forms with $m$ times differentiable coefficients as $C^{m} \Lambda^{k}$. In this work, we will only consider bounded spaces of continuous functions; thus, for functions in $C^{m} \Lambda^{k}$, the coefficients and their partial derivatives up to $m$-th order are taken to be finite (this space is sometimes referred to as $C_{B}^{m} \Lambda^{k}$, but we will omit the subscript $B$ ). Furthermore, under the weaker assumption that the coefficients of the alternating $k$-forms are integrable, given a basis for one-forms $\mu_{1} \ldots \mu_{d_{i}} \in \Lambda^{1}\left(\Omega_{i}\right)$, we can define the weighted inner product for $a, b \in \Lambda^{k}\left(\Omega_{i}\right)$,

$$
(a, b)_{\Omega_{i}}=\left(\sum_{\sigma} a_{\sigma} \mu_{\sigma(1)} \wedge \cdots \wedge \mu_{\sigma(k)}, \sum_{\sigma} b_{\sigma} \mu_{\sigma(1)} \wedge \cdots \wedge \mu_{\sigma(k)}\right)_{\Omega_{i}}=\int_{\Omega_{i}} \sum_{\sigma} a_{\sigma} b_{\sigma} \omega_{i},
$$

where $\sigma$ represents all ordered selections of $k$ values from $1 \ldots d_{i}$, and $\omega_{i} \in \Lambda^{d_{i}}\left(\Omega_{i}\right)$ is the unit volume form.

The vector spaces are pairwise dual to each other, in particular $\Lambda^{k} \sim \Lambda^{d_{i}-k}$. Dual forms are obtained by the Hodge star operator, such that $\star a \in \Lambda^{k}$ satisfies

$$
\int_{\Omega_{i}} a \wedge b=(\star a, b)_{\Omega_{i}} \quad \text { for all } b \in \Lambda^{k} .
$$

We choose the Euclidean metric in reference space $X_{i}$ as the underlying metric.

The inner product given in Eq. (2.4) induces a norm

$$
\|a\|=(a, a)^{1 / 2}
$$

and we define the spaces of square integrable forms

$$
L^{2} \Lambda^{k}:\left\{a \in \Lambda^{k} \mid\|a\|<\infty\right\} .
$$

For differentiable alternating forms, the exterior derivative d maps $\Lambda^{k} \rightarrow \Lambda^{k+1}$. We define the space of forms which have square integrable differentials as

$$
H \Lambda^{k}:\left\{a \in L^{2} \Lambda^{k} \mid \mathrm{d} a \in L^{2} \Lambda^{k+1}\right\} .
$$

These spaces are endowed with the norm $\|a\|_{\mathrm{d}}=\|a\|+\|\mathrm{d} a\|$. A proper subspace of the space $H \Lambda^{k}$ is that which includes natural boundary conditions with respect to the differential operator,

$$
\stackrel{\circ}{H} \Lambda^{k}:\left\{a \in H \Lambda^{k} \mid \operatorname{Tr} a=0\right\} .
$$

It is important to recall that the above spaces could equivalently be defined as the closure of $C^{\infty} \Lambda^{k}$ with respect to the stated norms [30].

By definition of the exterior derivative, the following sequence is a cochain complex, i.e., the differential operators map as shown

$$
0 \rightarrow \mathbb{R} \stackrel{\subset}{\rightarrow} H \Lambda^{0} \stackrel{\mathrm{d}}{\rightarrow} H \Lambda^{1} \stackrel{\mathrm{d}}{\rightarrow} \cdots \stackrel{\mathrm{d}}{\rightarrow} H \Lambda^{d} \rightarrow 0
$$

and $\operatorname{dd} a=0$ for all $a$.

In the case of $\partial Y=\partial_{D} Y$, the de Rham complex is extended by including the integral

$$
0 \rightarrow \stackrel{\circ}{H} \Lambda^{0} \stackrel{\mathrm{d}}{\rightarrow} \stackrel{\circ}{H} \Lambda^{1} \stackrel{\mathrm{d}}{\rightarrow} \cdots \stackrel{\mathrm{d}}{\rightarrow} \stackrel{\circ}{H} \Lambda^{d} \stackrel{\int}{\rightarrow} \mathbb{R} \rightarrow 0 .
$$


We refer to de Rham complexes such as (2.10) and (2.11) by the abbreviated notation $\left(H \Lambda^{\circ}, \mathrm{d}\right)$ and $\left(\stackrel{H}{H} \Lambda^{\bullet}, \mathrm{d}\right)$, respectively.

For contractible domains, the function spaces on alternating $k$-forms form an exact de Rham complex (extended in the sense of interpreting the inclusion of constant functions as a differential operator). Thus, every closed form (i.e., $\mathrm{d} a=0$ ) is exact (i.e., $a=\mathrm{d} b$ for some $b$ ). This is known as the Poincaré lemma. For general domains, the dimension of the cohomology space will be given by the Betti numbers.

In the case where $d=n=3$, the exterior derivative $\mathrm{d}$ corresponds to $\mathrm{d} \sim\{\nabla, \nabla \times, \nabla \cdot\}$ for the representatives of the $k$-forms. Furthermore, the spaces $H \Lambda^{k}$ correspond to the classical spaces $H \Lambda^{0} \sim H_{1}, H \Lambda^{1} \sim H(\nabla \times), H \Lambda^{2} \sim H(\nabla \cdot)$, and $H \Lambda^{3} \sim L^{2}$. The central part of the de Rham sequence (2.10) takes the form:

$$
H_{1} \stackrel{\nabla}{\longrightarrow} H(\nabla \times) \stackrel{\nabla \times}{\longrightarrow} H(\nabla \cdot) \stackrel{\nabla \cdot}{\longrightarrow} L^{2} .
$$

Note that the above definitions imply, from a formal perspective, that partial integration and Stokes' theorem are valid for $a \in H \Lambda^{k}$ and $b \in H \Lambda^{d_{i}-k-1}$ :

$$
\int_{\Omega_{i}} \mathrm{~d} a \wedge b+(-1)^{k} a \wedge \mathrm{d} b=\int_{\Omega_{i}} \mathrm{~d}(a \wedge b)=\int_{\partial \Omega_{i}} \operatorname{Tr}(a \wedge b)=\int_{\partial \Omega_{i}} \operatorname{Tr} a \wedge \operatorname{Tr} b .
$$

In order to rigorously show that the wedge product of traces is well defined in the final equality, a more careful treatment is needed. For the cases of primary interest in applications $(n \leq 3)$, Eq. (2.12) can be verified explicitly. Indeed, for $k=0$ the trace spaces are duals with respect to the boundary (resp. $H^{1 / 2} \Lambda^{0}$ and $H^{-1 / 2} \Lambda^{n-1}$ for the trace of $H \Lambda^{0}$ and $H \Lambda^{n-1}$ ), while the case of $n=3$ and $k=1$ has been analyzed separately [12]. We do not know of results which establish Eq. (2.12) rigorously for $n \geq 4$, although this result seems reasonable to conjecture. That said, our main motivation relates to physical problems, for which $n=3$, and we will therefore not elaborate further on this point.

We will need the codifferential operator, defined as the dual of the exterior derivative, which we denote by $\star \mathrm{d}^{*} a=(-1)^{k} \mathrm{~d} \star a$ for $a \in \Lambda^{k}$. The codifferential induces a function space

$$
H^{*} \Lambda^{k}:\left\{a \in L^{2} \Lambda^{k} \mid\left\|\mathrm{d}^{*} a\right\|<\infty\right\} .
$$

Using that $\star(\star a)=(-1)^{k\left(d_{i}+1\right)} a$, we can now write Stokes' theorem in terms of inner products: Let $a \in H \Lambda^{k}$, and $c \in H^{*} \Lambda^{k+1}$, then with $c=\star b$ we calculate (with $b \in H \Lambda^{d-k-1}$ ):

$$
(\mathrm{d} a, c)_{\Omega_{i}}-\left(a, \mathrm{~d}^{*} c\right)_{\Omega_{i}}=(-1)^{d_{i} k} \int_{\partial \Omega_{i}} \operatorname{Tr} a \wedge \operatorname{Tr} \star c=\left(\operatorname{Tr} a, \operatorname{Tr}^{*} c\right)_{\partial \Omega_{i}} .
$$

Here, we have introduced the dual trace (or cotrace) operator for $e \in \Lambda^{k}\left(\Omega_{i}\right)$ such that

$$
\star_{\partial} \operatorname{Tr}^{*} e=\operatorname{Tr}(\star e),
$$

where the $\star_{\partial}$ is the Hodge star with respect to the boundary. The dual trace does not appear to have standard notation, but appears, up to sign convention, in earlier works in various forms (see, e.g., [13, 21, 25] for related work). With our sign convention, the dual trace is commutative up to the codimension of the manifold,

$$
\operatorname{Tr}_{\partial_{j} \Omega_{i}}^{*} \mathrm{~d}^{*} e=(-1)^{d_{i}-d_{j}} \mathrm{~d}^{*} \operatorname{Tr}_{\partial_{j} \Omega_{i}}^{*} e .
$$


By applying (2.14) to $\mathrm{dd} a$, we obtain the following integration-by-parts formula on the boundary:

$$
\left(\operatorname{Tr} \mathrm{d} a, \operatorname{Tr}^{*} c\right)_{\partial \Omega_{i}}+\left(\operatorname{Tr} a, \operatorname{Tr}^{*} \mathrm{~d}^{*} c\right)_{\partial \Omega_{i}}=0
$$

For contractible domains, we have a Helmholtz decomposition, such that for all $a \in L^{2} \Lambda^{k}$, there exist $a_{\mathrm{d}} \in H \Lambda^{k}$ and $a_{\mathrm{d}^{*}} \in H^{*} \Lambda^{k}$ such that

$$
a=a_{\mathrm{d}^{*}}+a_{\mathrm{d}} \quad \text { while both } \quad \mathrm{d}^{*} a_{\mathrm{d}^{*}}=0=\mathrm{d} a_{\mathrm{d}} .
$$

For general domains, there may be a finite-dimensional cohomology, with dimension given by the Betti numbers, such that we have the Hodge decomposition

$$
a=a_{\mathrm{d}^{*}}+a_{\mathrm{d}}+a_{0} .
$$

In this case, the final term represents the cohomology class, and is a non-trivial solution to the equations $\mathrm{d} a_{0}=0=\mathrm{d}^{*} a_{0}$. For this decomposition to be unique, appropriate boundary conditions need to be imposed similar to those presented below in Theorem 3.4.

Finally, we recall the following form of the Poincaré-Friedrichs inequality: For $a \in \stackrel{\circ}{H} \Lambda^{k} \cap H^{*} \Lambda^{k}$ or $H \Lambda^{k} \cap \stackrel{\circ}{H}^{*} \Lambda^{k}$, it holds that

$$
\|a\|_{\Omega_{i}} \lesssim\|\mathrm{d} a\|_{\Omega_{i}}+\left\|\mathrm{d}^{*} a\right\|_{\Omega_{i}}+\left\|a_{0}\right\|_{\Omega_{i}} .
$$

The results stated above represent the main tools for developing elliptic differential equations, such as the Hodge Laplacian (that is to say, $\mathrm{dd}^{*}+\mathrm{d}^{*} \mathrm{~d}$ ), on manifolds. The main contribution of this paper is to extend these results and apply them in the setting of mixeddimensional geometries, as defined in Sect. 2.1.

\section{Differential forms}

In this section we provide an extension of the exterior derivative and the inner product to the geometry and structures of Sect. 2.1, and prove properties of the resulting operators. A main objective is to define function spaces on $\mathfrak{F}$ which retain the same structure as the classical function spaces of alternating forms on $Y$.

Semi-discrete differential operators appear in several applications. In addition to the references cited in the introduction, similar operators and structures to those defined in Sect. 3.1 have also recently been defined in order to consider mixed-type boundary conditions in the context of finite element exterior calculus [21].

\subsection{Mixed-dimensional $k$-forms}

We are interested in differential forms over $\Omega$ which preserve the properties known from Sect. 2.2. Let us therefore first define the integral over forests $\mathfrak{F}$, such that

$$
\int_{\mathfrak{F}} \mathfrak{w}=\sum_{j \in \mathscr{F}} \int_{\Omega_{s_{j}}} \omega_{j} .
$$

Here, we have introduced the mixed-dimensional volume form $\mathfrak{w}=\left[\omega_{j}\right]_{j \in \mathfrak{F}}$.

Recall that forms on $\Omega$ are defined with respect to $X$. That is, a form $a \in \Lambda^{k}\left(\Omega_{i}\right)$ exists if its pull-back $\phi_{0, i}^{*} a \in \Lambda^{k}\left(X_{i}\right)$. More generally, since $\phi$ is a coordinate system, statements such as 
" $a$ is integrable" are always understood to mean " $\phi^{*} a$ is integrable". The same holds for statements concerning continuity and differentiability. As an immediate example, this means that

$$
\int_{\Omega_{i}} a_{i} \equiv \int_{X_{i}} \phi_{0, i}^{*} a_{i} .
$$

Since each $\omega_{i} \in \Lambda^{d_{i}}\left(\Omega_{i}\right)$, we are motivated to define the mixed-dimensional space of $n$-forms on the forest $\mathfrak{F}$ as

$$
\mathfrak{Q}^{n}(\mathfrak{F})=\prod_{i \in I} \Lambda^{d_{i}}\left(\Omega_{i}\right)
$$

More generally, we are interested in extending alternating $k$-forms to the mixed-dimensional geometry. Consider therefore the following definition of alternating $k$-forms on a $\mathrm{DAG} \mathfrak{\Im}_{i}$ for $i \in I$,

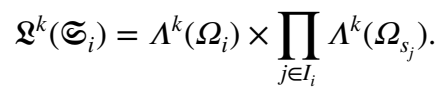

Here and in the following, we will use (without further comment) the convention that $\Lambda^{k}\left(\Omega_{i}\right)=0$ for $k \notin\left\{0,1, \ldots, d_{i}\right\}$. Note that with this convention, we observe that for $k=d_{i}$, only the root of the DAG contributes, i.e., $\mathfrak{Q}^{d_{i}}\left(\mathfrak{S}_{i}\right)=\Lambda^{d_{i}}\left(\Omega_{i}\right)$.

By assembling over all DAGs in a forest, we then obtain mixed-dimensional alternating $k$-forms on the full forest as

$$
\mathfrak{Q}^{k}(\mathfrak{F})=\prod_{i \in I} \mathfrak{Q}^{k-\left(n-d_{i}\right)}\left(\mathfrak{S}_{i}\right)
$$

It is clear that this is a generalization of the volume forms in the sense that we recover Eq. (3.3) from Eq. (3.5) for $k=n$. From Eq. (3.5), we note that for any root $i \in I$ and node $j \in \mathfrak{S}_{i}$, we denote the degree of the associated local form in $\mathfrak{Q}^{k}$ by $k_{j}=k-\left(n-d_{i}\right)$. It follows that $k_{j}$ only depends on the dimension of its root $i$, and not on $d_{j}$.

We will consistently use Gothic letters for mixed-dimensional functions and spaces on forests or DAGs, such as $\mathfrak{a} \in \mathfrak{Q}^{k}(\mathfrak{F})$, with the natural decomposition $\mathfrak{a}=\left\lfloor\mathfrak{a}_{i}\right\rfloor$ where $\mathfrak{a}_{i} \in \mathfrak{Q}^{k_{i}}\left(\mathfrak{S}_{i}\right)$. We use $l_{j}$ to denote the forms associated with node $j \in \mathfrak{F}$, such that $l_{j}: \mathfrak{Q}^{k}(\mathfrak{F}) \rightarrow \Lambda^{k_{j}}\left(\Omega_{s_{j}}\right)$. We will revert to regular Latin font for the fixed-dimensional alternating forms $a_{j}=\iota_{j} \mathfrak{a}$.

We will use three different spaces of forms on forests. These spaces generalize notions of square integrable functions, locally continuous functions, and weakly differentiable functions. First, we introduce $L^{2}$ functions over the mixed-dimensional structures.

Definition 3.1 Let the space of square integrable $k$-forms over $\mathfrak{F}$ be denoted $L^{2} \mathfrak{Q}^{k}(\mathfrak{F})$ and defined as

$$
L^{2} \mathfrak{Q}^{k}(\mathfrak{F}):\left\{\mathfrak{a} \in \mathfrak{Q}^{k}(\mathfrak{F}) \mid a_{j} \in L^{2} \Lambda^{k_{j}}\left(\Omega_{s_{j}}\right) \quad \forall j \in \mathfrak{F}\right\} .
$$

The space $L^{2} \mathfrak{Q}^{k}(\mathfrak{F})$ has an inner product defined for $\mathfrak{a}, \mathfrak{b} \in L^{2} \mathfrak{Q}^{k}(\mathfrak{F})$

$$
(\mathfrak{a}, \mathfrak{b})_{\mathfrak{F}}=\sum_{j \in \mathfrak{F}}\left(a_{j}, b_{j}\right)_{\Omega_{s_{j}}}
$$

and the inner product induces a norm on $L^{2} \mathfrak{Q}^{k}(\mathfrak{F})$ 


$$
\|\mathfrak{a}\|=(\mathfrak{a}, \mathfrak{a})_{\mathfrak{F}}^{1 / 2}
$$

Remark 3.1 The definition of the inner product naturally depends on the underlying metric. Since all $X_{i}$ are embedded in $\mathbb{R}^{d_{i}}$, we use the Euclidean metric in reference space.

Remark 3.2 The compact mixed-dimensional notation using the Gothic symbols greatly simplifies the exposition in the following sections. A concrete example of this will be evident in the applications in Sect. 4.3, where we provide the equivalence between elliptic PDEs stated in terms of mixed-dimensional and standard calculus notation.

As in the fixed-dimensional case, in order to treat degenerate coefficients that appear in different physical regimes, weighted inner products may be desirable in applications (for a more detailed discussion, see Sect. 4 and also [9]).

Lemma 3.1 The inner product defined in Eq. (3.6) is symmetric, linear, and positive-definite.

Lemma 3.2 The mixed-dimensional space $L^{2} \mathfrak{Q}^{k}(\mathfrak{F})$ is a Hilbert space.

Proof Due to the inner product (3.6), $L^{2} \mathfrak{Q}^{k}(\mathfrak{F})$ is pre-Hilbert. Completeness follows by the product structure given by Definition 3.1 .

\subsection{Strongly differentiable $\boldsymbol{k}$-forms}

We first define a notion of locally continuous forms.

Definition 3.2 Let the space of locally continuous mixed-dimensional $k$-forms over $\mathfrak{F}$ be denoted $C \mathbf{Q}^{k}(\mathfrak{F})$, defined such that

$$
\begin{aligned}
& C \mathfrak{Q}^{k}(\mathfrak{F}):\left\{\mathfrak{a} \in \mathfrak{Q}^{k}(\mathfrak{F}) \mid a_{j} \in C^{\infty} \Lambda^{k_{j}}\left(\Omega_{s_{j}}\right)\right. \text { and } \\
& \left.\quad a_{j}=\varepsilon\left(\Omega_{s_{j}}, \partial_{j} \Omega_{i}\right) \operatorname{Tr}_{\partial_{j} \Omega_{i}} a_{i} \quad \forall i \in I \text { and } j \in \mathfrak{S}_{i}\right\} .
\end{aligned}
$$

We denote by $\stackrel{\circ}{C} \mathfrak{Q}^{k}$ the subset of forms $\mathfrak{a} \in C \mathfrak{Q}^{k}$ such that $\operatorname{Tr} a_{i}=0$ on $\partial_{i} Y$.

Lemma 3.3 On each DAG $\mathfrak{S}_{i}$ with $i \in I$, the space $C \mathfrak{Q}^{k}\left(\mathfrak{S}_{i}\right)$ is isomorphic to $C^{\infty} \Lambda_{i}^{k}\left(\bar{X}_{i} \backslash \phi_{0, i}^{-1}\left(\partial_{i} Y\right)\right)$.

Proof Follows from Definition 2.1 and the defined maps $\phi_{0, i}[1]$.

Locally continuous forms are interpreted as (bounded) continuous $k_{i}$-forms on each $\Omega_{i}$, for $i \in I$, with continuous extensions onto the boundaries as appropriate. We provide three examples, two of which are illustrated for $n=1$ in Fig. 4.

Example 3.1 For $\mathfrak{a} \in C \mathbf{Q}^{0}(\mathfrak{F})$ and $i \in I^{n}$, we have that $a_{i}$ is a continuous, infinitely differentiable, function on $\Omega_{i}$. Discontinuities are permitted across all $\Omega_{j}$ for $j \in I^{d}$ where $d<n$, 
Fig. 4 Example of locally continuous functions in 1D. In this illustration, we have below the diagram indicated a domain decomposed into two 1D segments separated by a OD domain. The functions belonging to $C \mathbf{Q}^{0}$ and $C \mathbf{Q}^{n}$ are further described in Examples 3.1 and 3.3, respectively

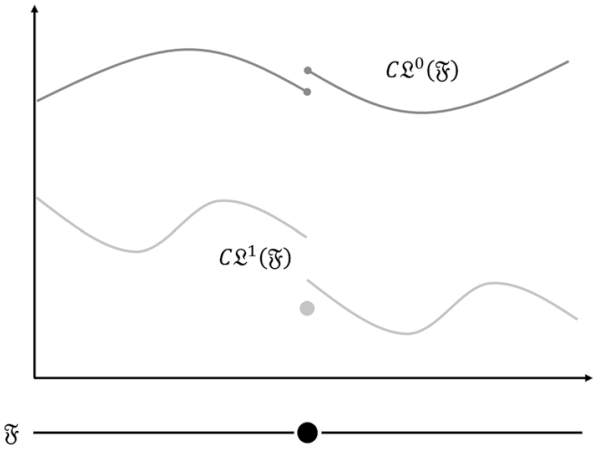

and the forms on $\Omega_{j}$ are void. In contrast, for all $l \in I_{i}$ the forms on $\partial_{l} \Omega_{i}$ are continuous and appropriately interpreted as traces of $a_{i}$ (up to sign).

Example 3.2 A form $\mathfrak{a} \in C \mathfrak{Q}^{n-1}(\mathfrak{F})$ can be represented by infinitely differentiable $d_{i}$-vector valued functions on all $\Omega_{i}$ with $d_{i}>0$. In Fig. 1, the box labeled $\mathfrak{Q}^{1}$ illustrates these as the roots $i \in\{3,4\}$. Moreover, the diagram shows that the local functions have infinitely differentiable (normal) traces on the descendants of codimension one, i.e., the boundary partitions corresponding to the four indices $j \in I_{3}^{0} \cup I_{4}^{1}$.

Example 3.3 The volume forms $\mathfrak{a} \in C \mathfrak{Q}^{n}(\mathfrak{F})$ are piecewise continuous functions, in the sense that $a_{i}$ are infinitely differentiable on each $\Omega_{i}$, for $i \in I$. Moreover, all $a_{i}$ become volume forms of the appropriate dimension. Thus, for all $l \in I_{i}$, the forms $a_{l}$ are void.

Theorem 3.1 The space $C \mathbf{Q}^{k}$ is dense in $L^{2} \mathbf{Q}^{k}$ with respect to the norm (3.7).

Proof We provide a constructive proof exploiting the product structure of $L^{2} \mathbf{Q}^{k}$. Let $\mathfrak{a} \in L^{2} \mathfrak{Q}^{k}$, and we will construct $\mathfrak{b} \in C \mathfrak{Q}^{k}$ such that $\|\mathfrak{a}-\mathfrak{b}\|<\epsilon$, for any $\epsilon>0$. Throughout the proof, we will work on the reference spaces $X_{i}$ in the sense of Sect. 2.1. Furthermore, let $\epsilon^{\prime}=\epsilon /|\mathfrak{|}|$, where $|\mathfrak{\Im}|$ is the number of nodes in $\mathfrak{F}$.

Consider first manifolds $j \in \mathfrak{F}$ such that $d_{j}=k_{j}$. Then, for all $j^{\prime} \in I_{j}, d_{j^{\prime}}<d_{j}=k_{j}=k_{j^{\prime}}$, and thus the local forms $a_{j^{\prime}}$ for $j^{\prime}$ are all void, while the local forms $a_{j}$ are in the usual spaces $L^{2} \Lambda^{k_{j}}\left(X_{j}\right)$. Since $C^{\infty} \Lambda^{k_{j}}\left(X_{j}\right)$ is dense in $L^{2} \Lambda^{k_{j}}\left(X_{j}\right)$, we can choose $b_{j} \in C^{\infty} \Lambda^{k_{j}}\left(X_{j}\right)$ such that $\left\|a_{j}-b_{j}\right\|_{X_{j}}<\epsilon^{\prime}$.

Proceeding recursively, let $j \in \mathfrak{F}$ such that $b_{j^{\prime}}$ is defined for all $j^{\prime} \in I_{j}$. Thus, since $\partial X=\bigcup_{j^{\prime} \in I_{j}} \phi_{j, j^{\prime}}\left(X_{j^{\prime}}\right)$, we can construct smooth extensions of the boundary data $b_{j^{\prime}}$ into $X_{j}$ [25]. Let $c_{j}$ be any such smooth extension, such that $\varepsilon\left(\Omega_{s_{j^{\prime}}}, \partial_{j^{\prime}} \Omega_{s_{j}}\right) \operatorname{Tr}_{\partial_{j^{\prime}} X_{j}} c_{j}=\phi_{j, j^{\prime}} b_{j^{\prime}}$. Then, we construct $a_{j}^{\prime}=a_{j}-c_{j} \in L^{2} \Lambda^{k_{j}}\left(X_{j}\right)$, and due to the density of $C_{0}^{\infty}$ in $L^{2}$, we can choose $b_{j}^{\prime} \in C_{0}^{\infty} \Lambda^{k_{j}}\left(X_{j}^{j}\right)$ such that $\left\|a_{j}^{\prime}-b_{j}^{\prime}\right\|_{X_{j}}<\epsilon^{\prime}$. We then define $b_{j}=b_{j}^{\prime}+c_{j} \in C^{\infty} \Lambda^{k_{j}}\left(X_{j}\right)$, and it follows trivially that $\left\|a_{j}-b_{j}\right\|_{X_{j}}<\epsilon^{\prime}$.

Finally, let $\mathfrak{b} \in \mathfrak{2}^{k}$ be defined such that $\iota_{j} \mathfrak{b}=b_{j}$. By construction, $\|\mathfrak{a}-\mathfrak{b}\|<\epsilon$, and it remains to verify that $\mathfrak{b} \in C \mathfrak{Q}^{k}$. With reference to Definition 3.2, this holds, since all ${ }_{l_{j}} \mathfrak{b} \in C^{\infty} \Lambda^{k_{j}}\left(X_{j}\right)$, and since $l_{j} \mathfrak{b}=\varepsilon\left(\Omega_{s_{j}}, \partial_{j} \Omega_{i}\right) \operatorname{Tr}_{\partial_{j} \Omega_{i}} l_{i} \mathfrak{b}$ by the construction above.

For continuous mixed-dimensional $k$-forms, we can define an appropriate exterior derivative in the same sense as $[21,27]$. 
Definition 3.3 For $\mathfrak{a} \in C \mathfrak{Q}^{k}(\mathfrak{F})$, let the strong form of the mixed-dimensional exterior derivative $\mathfrak{d}: C \mathfrak{Q}^{k}(\mathfrak{F}) \rightarrow C \mathfrak{Q}^{k+1}(\mathfrak{F})$ be defined for all $j \in \mathfrak{F}$ as

$$
l_{j}(\mathfrak{D a})=\mathrm{d} a_{j}+l_{j}(\mathbb{d} \mathfrak{a}),
$$

where the discrete differential operator $\mathbb{d}: C \mathfrak{Q}^{k}(\mathfrak{F}) \rightarrow C \mathfrak{Q}^{k+1}(\mathfrak{F})$ is defined on the roots $i \in I$ by

$$
l_{i}(\mathbb{d} \mathfrak{a})=(-1)^{n-k} \sum_{\left\{l \in \mathfrak{F} \mid s_{l}=i \text { and } \hat{l} \in I^{d_{i}+1}\right\}} \varepsilon\left(\Omega_{i}, \partial_{l} \Omega_{\hat{l}}\right) a_{l}
$$

and subsequently on all branches $j \in I_{i}$ as

$$
l_{j}(\mathbb{d} \mathfrak{a})=\varepsilon\left(\Omega_{s_{j}}, \partial_{j} \Omega_{i}\right) \operatorname{Tr}_{\partial_{j} \Omega_{i}} l_{i}(\mathbb{d} \mathfrak{a})
$$

Remark 3.3 This definition is equivalent to constructing a single graded complex from the anti-diagonals of the double complex induced by $\mathrm{d}$ and $\mathbb{d}$, as elaborated in [10]. In order to be self-contained, we provide the explicit construction here.

Due to the continuity properties of $C \mathbf{Q}^{k}$, the discrete operator $\mathbb{d}$ can be expressed locally for each node $j$ in the forest $\mathfrak{F}$. The local summation is then performed over an index set $\gamma_{j}^{-1}$ which we define below.

Corollary 3.1 For each $j \in \mathfrak{F}$, we can define a minimal set of indices, denoted $\gamma_{j}^{-1}$, such that for all $\mathfrak{a} \in C \mathfrak{Q}^{k}$, it holds that

$$
l_{j}(\mathbb{d} \mathfrak{a})=(-1)^{n-k} \sum_{l \in \gamma_{j}^{-1}} \varepsilon_{j l} a_{l}
$$

with $\varepsilon_{j l} \in\{ \pm 1\}$. More precisely, it follows from (3.9) and (3.10) that $\varepsilon_{j l}=\varepsilon\left(\Omega_{\hat{\jmath}}, \partial_{l^{\prime}} \Omega_{\hat{l}}\right)$ where the hat denotes the corresponding root and $l^{\prime}$ is the appropriate index in the set defined in (3.9).

Example 3.4 For all roots $i \in I$, we have $\gamma_{i}^{-1}=\left\{l \in \mathfrak{F} \mid s_{l}=i\right.$ and $\left.\hat{l} \in I^{d_{i}+1}\right\}$ as in (3.9), i.e., the set consists of all branches which geometrically coincide with $\Omega_{i}$ and have a root of dimension $d_{i}+1$. In the example from Fig. $1, \gamma_{3}^{-1}$ therefore consists of the indices $j_{3}, j_{4} \in I_{4}$ which have $s_{j_{3}}=s_{j_{4}}=3$.

Example 3.5 The set $\gamma_{j}^{-1}$ is void for branches $j \in I_{i}$ for which $l_{j}(\mathbb{d} \mathfrak{a})=0$ for all $\mathfrak{a} \in C \mathfrak{Q}^{k}$. An example of this arises in Fig. 1 where all $\mathfrak{a} \in C \mathbf{Q}^{0}$ map to zero at the extremities of $\Omega_{3}$, i.e., on the branches $j \in I_{3}$ with $d_{j}=0$.

The strong form of the mixed-dimensional exterior derivative is thus interpreted as the fixed-dimensional exterior derivative within each domain $X_{i}$, where the out-of-manifold components of the differential are expressed in terms of the traces of values on the manifolds which are in the neighborhood of $\Omega_{i}$. This definition is consistent with standard models for materials with thin inclusions [9, 15, 23]. Note that it is clear that the differential operator preserves continuity. 
In the absence of Dirichlet boundaries, i.e., $Y_{D}=\emptyset$, the mixed-dimensional spaces $C \mathbf{Q}^{k}$ as well as their relations given through the mixed-dimensional exterior derivative given in Definition 3.3, are summarized in the following diagram

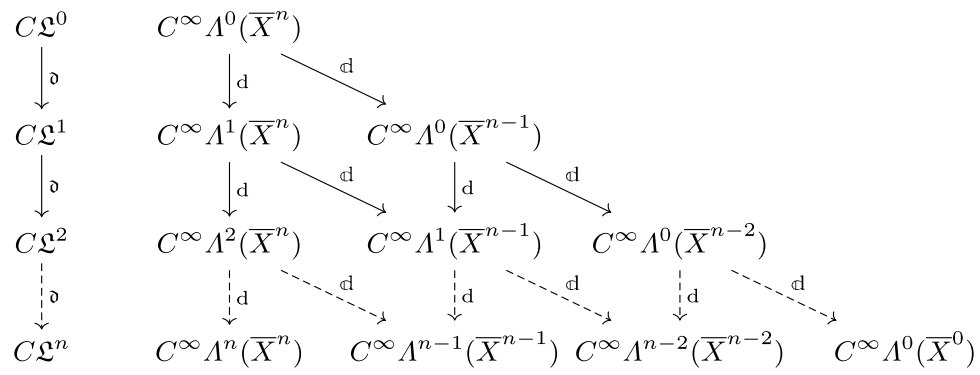

In order to provide this diagram, we have used the notation $X^{d}=\bigcup_{i \in I^{d}} X_{i}$. This diagram can be seen together with the forest in Fig. 1.

Lemma 3.4 The mixed-dimensional exterior derivative gives $C \mathfrak{Q}^{k}(\mathfrak{F})$ the structure of a cochain complex, i.e., $\mathfrak{d} \mathfrak{d}=0$ for all $\mathfrak{a} \in C \mathfrak{Q}^{k}(\mathfrak{F})$.

Proof By an explicit calculation, we have for arbitrary $j \in \mathfrak{F}$ :

$$
\iota_{j}\left(\mathfrak{d}^{2} \mathfrak{a}\right)=\mathrm{d}^{2} a_{j}+l_{j}(\mathrm{~d} \mathbb{d} \mathfrak{a})+l_{j}(\mathbb{d} \mathrm{d} \mathfrak{a})+l_{j}\left(\mathbb{d}^{2} \mathfrak{a}\right) .
$$

The first term is zero by the properties of the fixed-dimensional exterior derivative d. Additionally, the exterior derivative and the jump operator $\mathbb{d}$ are anticommutative:

$$
l_{j}(\mathrm{~d} \mathbb{d} \mathfrak{a})=\mathrm{d}\left((-1)^{n-k} \sum_{l \in \gamma_{j}^{-1}} \varepsilon_{j l} a_{l}\right)=-(-1)^{n-(k+1)} \sum_{l \in \gamma_{j}^{-1}} \varepsilon_{j l} l_{l}(\mathrm{~d} \mathfrak{a})=-l_{j}(\mathbb{d} \mathrm{d} \mathfrak{a}) ;
$$

hence, the second and third terms cancel.

Finally, the last term becomes

$$
l_{j}(\mathbb{d}(\mathbb{d} \mathfrak{a}))=(-1)^{n-k} \sum_{l \in \gamma_{j}^{-1}} \varepsilon_{j l} l_{l}(\mathbb{d} \mathfrak{a})=-\sum_{l \in \gamma_{j}^{-1}} \sum_{l^{\prime} \in \gamma_{l}^{-1}} \varepsilon_{j l} \varepsilon_{l l^{\prime}} a_{l^{\prime}}=0 .
$$

The last equality holds, since from the geometry, we see that each $a_{l^{\prime}}$ appears twice. The signs must be opposite depending on which intermediate manifold is used when taking boundary traces from $\Omega_{\hat{l}}$, thus for $l_{1}, l_{2} \in \gamma_{j}^{-1}$ with $l_{1} \neq l_{2}$ :

$$
\varepsilon_{j l_{1}} \varepsilon_{l_{1} l^{\prime}}=-\varepsilon_{j l_{2}} \varepsilon_{l_{2} l^{\prime}} \text {. }
$$

From the definitions and lemma above, the inclusion and mixed-dimensional exterior derivative $\mathfrak{d}$ lead to a de Rham complex $\left(C \mathfrak{Q}^{\bullet}, \mathfrak{d}\right)$ :

$$
0 \rightarrow \mathbb{R} \stackrel{\subset}{\rightarrow} C \mathbf{Q}^{0} \stackrel{\mathfrak{d}}{\rightarrow} C \mathfrak{Q}^{1} \stackrel{\mathfrak{d}}{\rightarrow} \cdots \stackrel{\mathfrak{d}}{\rightarrow} C \mathbf{Q}^{n} \rightarrow 0 .
$$


It will be of interest to have an explicit representation of a codifferential operator, which is consistent with an integration-by-parts formula with respect to the inner product (3.6). We therefore propose the following

Definition 3.4 For $\mathfrak{a} \in C \mathfrak{Q}^{k}(\mathfrak{F})$, let the strong form of the mixed-dimensional exterior coderivative $\mathfrak{d}^{*}: C \mathfrak{Q}^{k}(\mathfrak{F}) \rightarrow L^{2} \mathfrak{Q}^{k-1}(\mathfrak{F})$ be defined such that for all $\mathfrak{b} \in \stackrel{\circ}{C} \mathfrak{Q}^{k-1}(\mathfrak{F})$

$$
\left(\mathfrak{d}^{*} \mathfrak{a}, \mathfrak{b}\right)_{\mathfrak{F}}=(\mathfrak{a}, \mathfrak{d} \mathfrak{b})_{\mathfrak{F}} .
$$

Lemma 3.5 For $\mathfrak{a} \in C \mathfrak{Q}^{k}(\mathfrak{F})$, the strong form of the mixed-dimensional exterior coderivative has the explicit representation for all $j \in \mathfrak{F}$ as

$$
l_{j}\left(\mathfrak{D}^{*} \mathfrak{a}\right)=\mathrm{d}^{*} a_{j}+\sum_{l \in J_{j}^{d_{j}+1}} \operatorname{Tr}_{j}^{*} a_{l}+(-1)^{n-k} \sum_{l \in \gamma_{j}} \varepsilon_{l j} a_{l}
$$

with $\gamma_{j}=\left\{l \in \mathfrak{F} \mid j \in \gamma_{l}^{-1}\right\}$ and $J_{j}^{d_{j}+1}=\left\{l \in \mathfrak{F} \mid j \in I_{l}^{d_{l}-1}\right\}$.

Proof By definition of the mixed-dimensional exterior derivative and inner product, we calculate for $\mathfrak{b} \in \stackrel{\circ}{C} \mathfrak{Q}^{k-1}$

$$
\begin{aligned}
(\mathfrak{d} \mathfrak{b}, \mathfrak{a})_{\mathfrak{F}} & =\sum_{j \in \mathfrak{F}}\left(\mathrm{d} b_{j}+l_{j}(\mathbb{d} \mathfrak{b}), a_{j}\right)_{\Omega_{s_{j}}} \\
& =\sum_{j \in \mathfrak{F}}\left(\mathrm{d} b_{j}+(-1)^{n-k} \sum_{l \in \gamma_{j}^{-1}} \varepsilon_{j l} b_{l}, a_{j}\right)_{\Omega_{s_{j}}} \\
& =\sum_{j \in \mathfrak{F}}\left(b_{j}, \mathrm{~d}^{*} a_{j}\right)_{\Omega_{s_{j}}}+\sum_{l \in I_{j}^{d_{j}-1}}\left(\operatorname{Tr}_{l} b_{j}, \operatorname{Tr}_{l}^{*} a_{j}\right)_{\partial_{l} \Omega_{s_{j}}}+(-1)^{n-k} \sum_{l \in \gamma_{j}}\left(b_{j}, \varepsilon_{l j} a_{l}\right)_{\Omega_{s_{j}}} \\
& =\sum_{j \in \mathfrak{F}}\left(b_{j}, \mathrm{~d}^{*} a_{j}\right)_{\Omega_{s_{j}}}+\sum_{l \in J_{j}^{d_{j}+1}}\left(b_{j}, \operatorname{Tr}_{j}^{*} a_{l}\right)_{\Omega_{s_{j}}}+(-1)^{n-k} \sum_{l \in \gamma_{j}}\left(b_{j}, \varepsilon_{l j} a_{l}\right)_{\Omega_{s_{j}}} .
\end{aligned}
$$

It follows that the strong form proposed in Lemma 3.5 satisfies Definition 3.4. It remains to show uniqueness. Let $\mathfrak{c} \in L^{2} \mathfrak{Q}^{k-1}(\mathfrak{F})$ be any other codifferential of $\mathfrak{a}$. Then, by Definition 3.4

$$
\left(\mathfrak{c}-\mathfrak{d}^{*} \mathfrak{a}, \mathfrak{b}\right)_{\mathfrak{F}}=0
$$

for all $\mathfrak{b} \in \stackrel{\circ}{C}^{k-1}(\mathfrak{F})$. By the definition of the inner product, it then follows that $c_{i}-\imath_{i}\left(\mathfrak{D}^{*} \mathfrak{a}\right)$ is zero almost everywhere on $\Omega_{s_{i}}$ for all $i \in \mathfrak{F}$; thus, $\mathfrak{D}^{*} \mathfrak{a}$ is unique up to equivalence classes in $L^{2} \mathfrak{Q}^{k-1}(\mathfrak{F})$.

Remark 3.4 $\mathfrak{D}^{*} C \mathfrak{Q}^{k}$ has higher regularity than $L^{2} \mathfrak{Q}^{k-1}$, but we will not discuss this space further as it is not needed in this work.

The codifferential operator suggests the following definition of spaces of codifferentiable mixed-dimensional forms.

Definition 3.5 Let the space of codifferentiable mixed-dimensional $k$-forms over $\mathfrak{F}$, denoted by $C^{*} \mathbf{Q}^{k}(\mathfrak{F})$, be defined such that 


$$
C^{*} \mathfrak{Q}^{k}(\mathfrak{F}):\left\{\mathfrak{a} \in C \mathfrak{Q}^{k}(\mathfrak{F}) \mid \mathfrak{d}^{*} \mathfrak{a} \in C \mathfrak{Q}^{k-1}(\mathfrak{F})\right\} .
$$

We denote by $\stackrel{\circ}{C}^{*} \mathbf{Q}^{k}$ the subset of functions $\mathfrak{a} \in C^{*} \mathbf{Q}^{k}$ such that $\operatorname{Tr}^{*} a_{i}=0$ on $\partial_{i} Y$.

Lemma 3.6 For $\mathfrak{a} \in C^{*} \mathfrak{Q}^{k}(\mathfrak{F})$, it holds that $\mathfrak{b}^{*} \mathfrak{d}^{*} \mathfrak{a}=0$, and thus $\mathfrak{b}^{*}: C^{*} \mathfrak{Q}^{k}(\mathfrak{F}) \rightarrow C^{*} \mathfrak{Q}^{k-1}(\mathfrak{F})$.

Proof The statement that $\mathfrak{d}^{*} \mathfrak{d}^{*} \mathfrak{a}=0$ follows by applying Definition 3.4 twice, and using that $\mathfrak{d} \mathfrak{d}=0$. The second statement follows since $0 \in C \mathfrak{Q}^{k-2}(\mathfrak{F})$, thus $\mathfrak{D}^{*} \mathfrak{a} \in C^{*} \mathfrak{Q}^{k-1}(\mathfrak{F})$.

The codifferential operator and spaces also form a de Rham complex $\left(C^{*} \mathbf{Q}^{*}, \mathfrak{d}^{*}\right)$ :

$$
0 \leftarrow C^{*} \mathbf{Q}^{0} \stackrel{\mathfrak{d}^{*}}{\longleftarrow} C^{*} \mathbf{Q}^{1} \stackrel{\mathfrak{d}^{*}}{\longleftarrow} \cdots \stackrel{\mathfrak{d}^{*}}{\longleftarrow} C^{*} \mathbf{Q}^{n} \stackrel{\supset}{\longleftarrow} \mathbb{R} \leftarrow 0 .
$$

The de Rham complexes with boundary conditions $\left(\stackrel{\circ}{C} \mathfrak{Q}^{\bullet}, \mathfrak{d}\right)$ and $\left(\stackrel{\circ}{C}^{*} \mathfrak{Q}^{*}, \mathfrak{d}^{*}\right)$ are defined similarly, but with the integral instead of the inclusions, equivalent to (2.10) and (2.11).

Remark 3.5 The forms $\mathfrak{a} \in C^{*} \mathfrak{Q}^{k}(\mathfrak{F})$ satisfy, in addition to the conditions of Definition 3.2, also the analogous conditions related to the codifferential, i.e., that for all $i \in I$ and $j \in I_{i}$

$$
l_{j}\left(\mathfrak{D}^{*} \mathfrak{a}\right)=\varepsilon\left(\Omega_{s_{j}}, \partial_{j} \Omega_{i}\right) \operatorname{Tr}_{\partial_{j} \Omega_{i}} l_{i}\left(\mathfrak{D}^{*} \mathfrak{a}\right) .
$$

Using Lemma 3.5, this provides an explicit constraint on the form $\mathfrak{a}$ in the sense that

$$
\operatorname{Tr}_{\partial_{j} \Omega_{i}} \mathrm{~d}^{*} a_{i}=\varepsilon\left(\Omega_{s_{j}}, \partial_{j} \Omega_{i}\right)\left[\mathrm{d}^{*} a_{j}+\sum_{l \in J_{j}^{d_{j}+1}} \operatorname{Tr}_{j}^{*} a_{l}+(-1)^{n-k} \sum_{l \in \gamma_{j}} \varepsilon_{l j} a_{l}\right] .
$$

\subsection{Weak differentials and Hilbert spaces}

In order to provide a suitable framework for working with partial differential equations, we are also interested in weak forms of the function spaces and operators introduced above.

Definition 3.6 For $\mathfrak{a} \in L^{2} \mathfrak{Q}^{k}(\mathfrak{F})$, with $a_{j} \in H \Lambda^{k_{j}}\left(\Omega_{s_{j}}\right)$ for all $j \in \mathfrak{F}$, let a weak mixeddimensional exterior derivative of $\mathfrak{a}$, if it exists, be any form $\mathfrak{d} \mathfrak{a} \in L^{2} \mathfrak{Q}^{k+1}(\mathfrak{F})$ such that for all $\mathfrak{b} \in \stackrel{\circ}{C} \mathfrak{Q}^{k+1}(\mathfrak{F})$

$$
(\mathfrak{d} \mathfrak{a}, \mathfrak{b})_{\mathfrak{F}}=\left(\mathfrak{a}, \mathfrak{b}^{*} \mathfrak{b}\right)_{\mathfrak{F}}
$$

Definition 3.7 We denote the space of weakly differentiable mixed-dimensional forms on the forest $\mathfrak{F}$ as

$$
H \mathfrak{Q}^{k}(\mathfrak{F}):\left\{\mathfrak{a} \in L^{2} \mathfrak{Q}^{k}(\mathfrak{F}) \mid \mathfrak{d a} \in L^{2} \mathfrak{Q}^{k+1}(\mathfrak{F})\right\}
$$

We allow for boundary conditions on the external boundary $\partial_{D} Y$ in the sense of

$$
\stackrel{\circ}{H \mathfrak{Q}^{k}}(\mathfrak{F}):\left\{\mathfrak{a} \in H \mathfrak{Q}^{k}(\mathfrak{F}) \mid \operatorname{Tr}_{\partial_{i} Y} a_{i}=0 \text { for all } i \in \mathfrak{F}\right\} .
$$

For both $H \mathbf{Q}^{k}$ and $\stackrel{\circ}{H} \mathbf{Q}^{k}$ the natural norm is given as 


$$
\|\mathfrak{a}\|_{\mathfrak{b}}=\|\mathfrak{a}\|+\|\mathfrak{d} \mathfrak{a}\|
$$

Lemma 3.7 The mixed-dimensional spaces $H \mathfrak{Q}^{k}(\mathfrak{F})$ and $\stackrel{H}{H} \mathfrak{Q}^{k}(\mathfrak{F})$ are Hilbert spaces.

Proof The case $k=n$ is immediate from Lemma 3.2, as $H \mathfrak{Q}^{n}(\mathfrak{F})=L^{2} \mathfrak{Q}^{n}(\mathfrak{F})$. For the general case, consider any Cauchy sequence $\mathfrak{a}_{l} \in H \mathfrak{\Omega}^{k}(\mathfrak{F})$. Then, due to the completeness of $L^{2} \mathfrak{Q}^{k}(\mathfrak{F})$, the limits $\overline{\mathfrak{a}}=\lim _{l \rightarrow \infty} \mathfrak{a}_{l}$ and $\overline{\mathfrak{d} \mathfrak{a}}=\lim _{l \rightarrow \infty} \mathfrak{d \mathfrak { a } _ { l }}$ exist. It remains to show that $\overline{\mathfrak{b} \mathfrak{a}}=\mathfrak{b} \overline{\mathfrak{a}}$. This holds by a standard calculation, since for any $\mathfrak{c} \in \stackrel{\circ}{\mathcal{C}} \mathfrak{Q}^{k+1}(\mathfrak{F})$,

$$
(\overline{\mathfrak{d a}}, \mathfrak{c})_{\mathfrak{F}}=\lim _{l \rightarrow \infty}\left(\mathfrak{d} \mathfrak{a}_{l}, \mathfrak{c}\right)_{\mathfrak{F}}=\lim _{l \rightarrow \infty}\left(\mathfrak{a}_{l}, \mathfrak{b}^{*} \mathfrak{c}\right)_{\mathfrak{F}}=\left(\overline{\mathfrak{a}}, \mathfrak{b}^{*} \mathfrak{c}\right)_{\mathfrak{F}}=(\mathfrak{d} \overline{\mathfrak{a}}, \mathfrak{c})_{\mathfrak{F}} .
$$

The same calculation holds for $\stackrel{\circ}{H}^{k}(\mathfrak{F})$ when $C \mathfrak{Q}^{k+1}(\mathfrak{F})$ is used as the space of test functions.

In order to provide a characterization of $H \mathbf{Q}^{k}(\mathfrak{F})$ in terms of local spaces on each $\Omega_{i}$, we introduce a subspace of the standard space $H \Lambda^{k}$. Thus, for each root $i \in I$, we consider functions $a_{i}$ from the space of weakly differentiable $k_{i}$-forms subject to boundary conditions imposed by the forms $a_{j}$ for all $j \in I_{i}$. Locally, we refer to these spaces with imposed trace regularity using the recursive definition

$$
H \Lambda^{k}\left(\Omega_{i}, \operatorname{Tr}\right)=\left\{a \in H \Lambda^{k}\left(\Omega_{i}\right) \mid \operatorname{Tr}_{\partial_{j} \Omega_{i}} a \in H \Lambda^{k}\left(\Omega_{s_{j}}, \operatorname{Tr}\right) \forall j \in I_{i}\right\}
$$

with a naturally induced, recursively defined, norm

$$
\|a\|_{H \Lambda^{k}\left(\Omega_{i}, \operatorname{Tr}\right)}=\|a\|_{H \Lambda^{k}\left(\Omega_{i}\right)}+\sum_{j \in I_{i}}\left\|\operatorname{Tr}_{\partial_{j} \Omega_{s_{i}}} a\right\|_{H \Lambda^{k}\left(\Omega_{s_{j}}, \operatorname{Tr}\right)} .
$$

Example 3.6 For $k=d_{i}$, the space $H \Lambda^{k}\left(\Omega_{i}\right.$, Tr $)$ corresponds to the classical space $L^{2}\left(\Omega_{i}\right)$.

Example 3.7 For $d_{i} \geq 2$ and $k=d_{i}-1$, the space $H \Lambda^{k}\left(\Omega_{i}\right.$, Tr ) corresponds to the subspace of the classical space $H\left(\nabla \cdot ; \Omega_{i}\right)$ with the restriction that its traces are square integrable. It can thus be described by

$$
H \Lambda^{d_{i}-1}\left(\Omega_{i}, \operatorname{Tr}\right)=\left\{a_{i} \in H\left(\nabla \cdot ; \Omega_{i}\right) \mid \operatorname{Tr}_{\partial_{j} \Omega_{i}} a_{i} \in L^{2}\left(\Omega_{s_{j}}\right) \forall j \in I_{i}^{d_{i}-1}\right\} .
$$

This is in contrast to the full space $H\left(\nabla \cdot ; \Omega_{i}\right)$, which contains function traces with only $H^{-1 / 2}$ regularity. Although there is no common notation for spaces with enhanced boundary regularity, they appear in applications, see, e.g., $[9,16,23]$.

Lemma 3.8 There exist bounded extension operators $\mathcal{R}: H \Lambda^{k}\left(\partial X_{i}\right) \rightarrow H \Lambda^{k}\left(X_{i}\right)$.

Proof See, e.g., [25].

Lemma $3.9 H \Lambda^{k}\left(\Omega_{i}, \operatorname{Tr}\right)$ is a Hilbert space.

Proof Consider $i \in I, j \in I_{i}$, and let first $k=d_{i}-1$. Then, $\phi_{i, j}\left(X_{j}\right)$ is a smooth $d_{j}$-dimensional subset of the boundary of $X_{i}$ for each $j \in I_{i}^{d_{i}-1}$, and $H \Lambda^{k}\left(X_{j}\right)=L^{2} \Lambda^{k}\left(X_{j}\right)$. Using the extension operator from Lemma 3.8, we obtain 


$$
H \Lambda^{k}\left(X_{i}, \operatorname{Tr}\right)=\stackrel{\circ}{H} \Lambda^{k}\left(X_{i}\right) \times \Pi_{j \in I_{i}^{d_{i}-1}} \mathcal{R} \circ \phi_{i, j}\left(H \Lambda^{k}\left(X_{j}\right)\right) .
$$

Due to the product structure, $H \Lambda^{k}\left(X_{i}, \operatorname{Tr}\right)$ is therefore complete.

For $k<d_{i}-1$, the same argument is applied recursively.

Next, we observe that these local spaces allow for the decomposition of $H \mathbf{\Omega}^{k}$ into a product structure similar to that derived for $L^{2} \mathbf{Q}^{k}$ in Definition 3.1. In particular, we obtain the following, alternative characterization of $H \mathbf{Q}^{k}$ :

$$
\begin{aligned}
& H \mathfrak{Q}^{k}(\mathfrak{F}):\left\{\mathfrak{a} \in \mathfrak{Q}^{k}(\mathfrak{F}) \mid a_{i} \in H \Lambda^{k_{i}}\left(\Omega_{i}, \operatorname{Tr}\right)\right. \\
& \left.\quad \text { and } a_{j}=\varepsilon\left(\Omega_{s_{j}}, \partial_{j} \Omega_{i}\right) \operatorname{Tr}_{\partial_{j} \Omega_{i}} a_{i} \quad \forall i \in I \text { and } j \in I_{i}\right\}
\end{aligned}
$$

Lemma 3.10 Definition 3.7 and (3.31) are equivalent.

Proof Within this proof, we denote the space defined in Definition 3.7 as $H_{1} \mathfrak{Q}^{k}(\mathfrak{F})$ and the space defined in Eq. (3.31) as $H_{2} \mathfrak{Q}^{k}(\mathfrak{F})$.

Suppose $\mathfrak{a} \in H_{1} \mathfrak{Q}^{k}(\mathfrak{F})$. By the same calculation as in the proof of Lemma 3.5, we obtain that

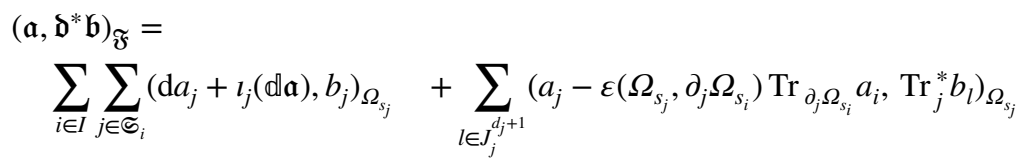

for all $\mathfrak{b} \in \stackrel{\circ}{C} \mathfrak{Q}^{k+1}(\mathfrak{F})$. Since the second term cannot be represented in $\mathfrak{Q}^{k+1}(\mathfrak{F}$ ) (as it contains an inner product of forms in $\mathfrak{Q}^{k}(\mathfrak{F})$ ), it must be zero for $\mathfrak{d} \mathfrak{a}$ to exist. However, since $\mathfrak{b}$ is arbitrary and $C \Lambda^{k_{i}}\left(\Omega_{j}\right)$ is dense in $L^{2} \Lambda^{k_{i}}\left(\Omega_{j}\right)$, the continuity condition in Eq. (3.31) is a necessary consequence of Definition 3.7. Moreover, we note that as in the strong case, the weak mixed-dimensional differential can be expressed locally by the weak differentials as

$$
l_{j}(\mathfrak{d a})=\mathrm{d} a_{j}+l_{j}(\mathbb{d} \mathfrak{a}) .
$$

Again, by the density of $C \Lambda^{k_{i}}\left(\Omega_{j}\right)$, it follows that the existence of $l_{j} \mathfrak{d a}$ implies $a_{j} \in H \Lambda^{k_{j}}\left(\Omega_{s_{j}}\right)$ for all $j \in \mathfrak{F}$, and therefore $H_{1} \mathfrak{2}^{k}(\mathfrak{F}) \subseteq H_{2} \mathfrak{Q}^{k}(\mathfrak{F})$.

Conversely, suppose $\mathfrak{a} \in H_{2} \mathfrak{Q}^{k}(\mathfrak{F})$. Then, the right-hand side of Eq. (3.33) is well defined, and we can construct $\mathfrak{d a}$. It remains to show that

$$
\|\mathfrak{d a}\|_{\mathfrak{D}}<\infty \text {. }
$$

However, this is a direct consequence of the definition of the norm and inner products, Eqs. (3.6) and (3.7). Therefore, also $H_{2} \mathfrak{Q}^{k}(\mathfrak{F}) \subseteq H_{1} \mathfrak{Q}^{k}(\mathfrak{F})$, and the Lemma is proved.

As a direct result of Lemma 3.10, we have a characterization of $H \mathbf{Q}^{k}$ in terms of local spaces. These can be organized in the following diagram, analogous to (3.16). Note that only the bottom row of spaces, containing $d$-forms on $d$-manifolds, does not have increased trace regularity. 


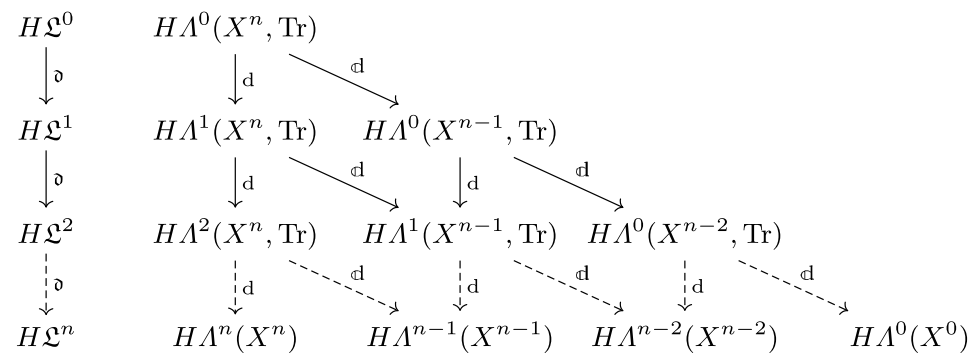

Corollary 3.2 The space $C \mathfrak{Q}^{k}$ is dense in $H \mathfrak{Q}^{k}$ with respect to the norm (3.25).

Proof Lemma 3.10 shows that the space $H \mathbf{Q}^{k}$ is isomorphic to the product space $\Pi_{i \in I} H \Lambda^{k_{i}}\left(\Omega_{i}, \operatorname{Tr}\right)$. Furthermore, Eq. (3.30) in Lemma 3.9 shows that each local space $H \Lambda^{k}\left(\Omega_{j}, \operatorname{Tr}\right)$ also enjoys a product structure. Each of the factors in this product structure is isomorphic to $\stackrel{\circ}{H} \Lambda^{k_{j}}\left(X_{j}\right)$, for some $j$. Since $C^{\infty} \Lambda^{k_{j}}\left(X_{j}\right)$ is dense in these spaces, the corollary follows.

A similar construction leads us to the weak codifferential operators and corresponding spaces.

Definition 3.8 For $\mathfrak{a} \in L^{2} \mathfrak{Q}^{k}(\mathfrak{F})$, with $a_{i} \in H^{*} \Lambda^{k_{i}}\left(\Omega_{s_{i}}\right.$ ) for all $i \in \mathfrak{F}$, let a weak mixeddimensional exterior coderivative of $\mathfrak{a}$, if it exists, be any form $\mathfrak{d}^{*} \mathfrak{a} \in L^{2} \mathfrak{Q}^{k-1}(\mathfrak{F})$ such that for all $\mathfrak{b} \in \stackrel{\circ}{H} \mathfrak{Q}^{k-1}(\mathfrak{F})$,

$$
\left(\mathfrak{d}^{*} \mathfrak{a}, \mathfrak{b}\right)_{\mathfrak{F}}=(\mathfrak{a}, \mathfrak{d} \mathfrak{b})_{\mathfrak{F}}
$$

Definition 3.9 We denote the space of weakly codifferentiable mixed-dimensional forms on the forest $\mathfrak{F}$ as

$$
H^{*} \mathfrak{Q}^{k}:\left\{\mathfrak{a} \in L^{2} \mathfrak{Q}^{k} \mid \mathfrak{b}^{*} \mathfrak{a} \in L^{2} \mathfrak{Q}^{k-1}\right\} .
$$

We allow for boundary conditions on the external boundary $\partial_{D} Y$ in the sense of

$$
\stackrel{\circ}{H}^{*} \mathfrak{Q}^{k}:\left\{\mathfrak{a} \in H \mathfrak{Q}^{k} \mid \operatorname{Tr}_{\partial_{i} Y}^{*} a_{i}=0 \text { for all } i \in \mathfrak{F}\right\} .
$$

For both $H^{*} \mathbf{Q}^{k}$ and $\stackrel{\circ}{H}^{*} \mathbf{Q}^{k}$ the natural norm is given as

$$
\|\mathfrak{a}\|_{\mathfrak{D}^{*}}=\|\mathfrak{a}\|+\left\|\mathfrak{D}^{*} \mathfrak{a}\right\| .
$$

The same considerations as elaborated above for $H \mathfrak{Q}^{k}(\mathfrak{F})$ can be extended to $H^{*} \mathfrak{Q}^{k}(\mathfrak{F})$, and we summarize these without proof:

Corollary 3.3 The mixed-dimensional spaces $H^{*} \mathbf{Q}^{k}(\mathfrak{F})$ and $\stackrel{\circ}{H}^{*} \mathbf{Q}^{k}(\mathfrak{F})$ are Hilbert spaces, and the space $C^{*} \mathbf{Q}^{k}(\mathfrak{F})$ is dense in $H^{*} \mathbf{Q}^{k}(\mathfrak{F})$ with respect to the norm (3.38). 


\subsection{Stokes' theorem and Poincaré lemma}

We verify that standard tools are available in the mixed-dimensional setting, starting with Stokes' theorem.

Theorem 3.2 (Stokes') For any $\mathfrak{a} \in H \mathfrak{Q}^{n-1}(\mathfrak{F})$ it holds that

$$
\int_{\mathfrak{F}} \mathfrak{d} \mathfrak{a}=\sum_{i \in I} \int_{\partial_{i} Y} \operatorname{Tr}_{\partial_{i} Y} a_{i}
$$

Proof Since $\mathfrak{d a} \in H \mathfrak{Q}^{n}$ is defined only on the roots, an explicit calculation gives

$$
\int_{\mathfrak{F}} \mathfrak{d} \mathfrak{a}=\sum_{i \in I} \int_{\Omega_{i}} \mathrm{~d} a_{i}+l_{i}(\mathbb{d} \mathfrak{a})=\sum_{i \in I} \int_{\partial \Omega_{i}} \operatorname{Tr}_{\partial \Omega_{i}} a_{i}-\int_{\Omega_{i}} \sum_{l \in \gamma_{i}^{-1}} \varepsilon_{i l} a_{l}=\sum_{i \in I} \int_{\partial_{i} Y} \operatorname{Tr}_{\partial_{i} Y} a_{i} .
$$

Stokes' theorem allows us to show that the mixed-dimensional exterior derivative is in a certain sense the correct generalization of the exterior derivative on $Y$ to $\Omega$. As indicated by diagram (3.11), the integral and exterior derivative $\mathfrak{D}$ defined in Sect. 3.1 lead to a de Rham complex $\left(H \mathfrak{Q}^{\bullet}, \mathfrak{D}\right)$ :

$$
0 \rightarrow \mathbb{R} \stackrel{\subset}{\rightarrow} H \mathfrak{Q}^{0} \stackrel{\mathfrak{d}}{\rightarrow} H \mathbf{\Omega}^{1} \stackrel{\mathfrak{d}}{\rightarrow} \cdots \stackrel{\mathfrak{d}}{\rightarrow} H \mathbf{Q}^{n} \rightarrow 0 .
$$

Moreover, with boundary conditions imposed on $\partial_{D} Y$, Theorem 3.2 gives us the mixeddimensional analogue of (2.11)

$$
0 \stackrel{\subset}{\rightarrow} \stackrel{\circ}{H} \mathbf{Q}^{0} \stackrel{\mathfrak{d}}{\rightarrow} \stackrel{\circ}{H} \mathbf{Q}^{1} \stackrel{\mathfrak{d}}{\rightarrow} \cdots \stackrel{\mathfrak{d}}{\rightarrow} \stackrel{\circ}{H} \mathbf{Q}^{n} \stackrel{\int_{\mathfrak{F}}}{\longrightarrow} \mathbb{R} \rightarrow 0 .
$$

Next, we consider the cohomology classes of (3.41) in the following theorem.

Theorem 3.3 For $n \leq 3$, the dimensions of the cohomology spaces of the cochain complex $\left(H \mathbf{Q}^{\bullet}(\mathfrak{F}), \mathfrak{d}\right)$ are equal to those of the complex $\left(H \Lambda^{\bullet}(Y), \mathrm{d}\right)$.

Proof The proof follows the double-complex construction of [10, 34]. To verify all the steps in this construction in the case of mixed-dimensional differential forms, a detailed exposition is presented in Appendix.

The de Rham complex with boundary conditions $\left(\stackrel{\circ}{H} \mathfrak{Q}^{\bullet}, \mathfrak{D}\right)$ is defined similar to (3.41).

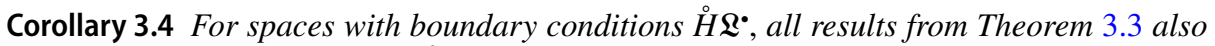
hold for the de Rham complex $\left(\stackrel{\circ}{H} \mathfrak{Q}^{\bullet}, \mathfrak{d}\right)$.

Proof Since the trace on $\partial Y$ commutes with the (mixed-dimensional) exterior derivative, the result is immediate.

The codifferential also defines de Rham complexes $\left(H^{*} \mathfrak{Q}^{\bullet}, \mathfrak{d}^{*}\right)$ and $\left(\stackrel{\circ}{H}^{*} \mathfrak{Q}^{\bullet}, \mathfrak{d}^{*}\right)$, similar to $(3.21)$. 
Corollary $3.5\left(H^{*} \mathfrak{Q}^{\bullet}, \mathfrak{d}^{*}\right)$ and $\left(\stackrel{H}{*}^{*} \mathfrak{Q}^{\bullet}, \mathfrak{D}^{*}\right)$ are chain complexes, i.e., for all $\mathfrak{a} \in H^{*} \mathbf{Q}^{k}$, $\mathfrak{d}^{*} \mathfrak{d}^{*} \mathfrak{a}=0$.

Proof For any $\mathfrak{a} \in H^{*} \mathfrak{Q}^{k}$, application of integrating by parts twice gives:

$$
\left(\mathfrak{d}^{*} \mathfrak{d}^{*} \mathfrak{a}, \mathfrak{b}\right)_{\mathfrak{F}}=\left(\operatorname{Tr} \mathfrak{d} \mathfrak{b}, \operatorname{Tr}^{*} \mathfrak{a}\right)_{\partial Y}+\left(\operatorname{Tr} \mathfrak{b}, \operatorname{Tr}^{*} \mathfrak{d}^{*} \mathfrak{a}\right)_{\partial Y} \quad \text { for all } \mathfrak{b} \in H \mathfrak{Q}^{k-2}
$$

Thus, $\left(\mathfrak{d}^{*} \mathfrak{d}^{*} \mathfrak{a}, \mathfrak{b}\right)=0$ for all $\mathfrak{a} \in \stackrel{\circ}{H}^{*} \mathfrak{Q}^{k}$, and it follows that $\mathfrak{b}^{*} \mathfrak{D}^{*} \mathfrak{a}=0$ pointwise away from the boundary. But then since the boundary has no measure it follows that $\left(\mathfrak{d}^{*} \mathfrak{d}^{*} \mathfrak{a}, \mathfrak{b}\right)=0$ for all $\mathfrak{a}$. As a consequence, we obtain the generalization of the integration by parts formula on the boundary

$$
\left(\operatorname{Tr} \mathfrak{d} \mathfrak{b}, \operatorname{Tr}^{*} \mathfrak{a}\right)_{\partial Y}+\left(\operatorname{Tr} \mathfrak{b}, \operatorname{Tr}^{*} \mathfrak{b}^{*} \mathfrak{a}\right)_{\partial Y}=0 \quad \text { for all } \mathfrak{a} \in H^{*} \mathfrak{Q}^{k} \text { and } \mathfrak{b} \in H \mathfrak{Q}^{k-2} .
$$

Remark 3.6 We expect the constraint $n \leq 3$ in Theorem 3.3 to be superfluous. However, we have not confirmed all details for $n \geq 4$.

The cohomology structure of the complexes from Corollary 3.5 will be considered in the following subsection.

\subsection{Helmholtz and Hodge decompositions}

The Helmholtz and Hodge decompositions of the standard Sobolev spaces extend to the mixed-dimensional setting.

Theorem 3.4 For any $\mathfrak{a} \in L^{2} \mathfrak{Q}^{k}$, there exist a Hodge decomposition $\mathfrak{a}_{\mathfrak{d}} \in H \mathfrak{Q}^{k}, \mathfrak{a}_{\mathfrak{b}^{*}} \in \stackrel{H}{H}^{*} \mathfrak{Q}^{k}$ and $\mathfrak{a}_{0} \in \mathfrak{H}^{k}$ such that

$$
\mathfrak{a}=\mathfrak{a}_{\mathfrak{b}}+\mathfrak{a}_{\mathfrak{b}^{*}}+\mathfrak{a}_{0}
$$

while $\mathfrak{D}^{*} \mathfrak{a}_{\mathfrak{b}^{*}}=\mathfrak{d} \mathfrak{a}_{\mathfrak{b}}=\mathfrak{d}^{*} \mathfrak{a}_{0}=\mathfrak{d} \mathfrak{a}_{0}=0$. The space $\mathfrak{H}^{k}$ is referred to as the space of mixeddimensional harmonic forms, is isomorphic to the cohomology, and is defined by

$$
\mathfrak{S}^{k}=\left\{\mathfrak{c} \in H \mathfrak{Q}^{k} \cap \stackrel{\circ}{H}^{*} \mathfrak{Q}^{k} \mid \mathfrak{d}^{*} \mathfrak{c}=\mathfrak{d} \mathfrak{c}=0\right\} .
$$

On contractible domains, $\mathfrak{S}^{k}=0$ and $\mathfrak{a}_{0}=0$, and we refer to the remaining two terms as the Helmholtz decomposition.

Proof We introduce the notation $\mathfrak{D} H \mathfrak{Q}^{k} \subset H \mathfrak{Q}^{k+1}$ and $\mathcal{N}\left(H \mathfrak{Q}^{k}, \mathfrak{D}\right) \subset H \mathfrak{\Omega}^{k}$ to indicate the range and null-space of $\mathfrak{d}$, respectively. Due to the integration-by-parts formula Eq. (3.35), it is clear that the space $L^{2} \mathfrak{Q}^{k}$ decomposes into orthogonal complements

$$
\mathfrak{D} H \mathfrak{\Omega}^{k-1} \perp \mathcal{N}\left(\stackrel{\circ}{H}^{*} \mathfrak{Q}^{k}, \mathfrak{D}^{*}\right),
$$

where $\mathcal{N}\left(\stackrel{\circ}{H}^{*} \mathfrak{Q}^{k}, \mathfrak{d}^{*}\right) \supseteq \mathfrak{d}^{*} \stackrel{\circ}{H}^{*} \mathfrak{Q}^{k+1}$. Thus, we obtain

$$
L^{2} \mathfrak{Q}^{k}=\mathfrak{d} H \mathfrak{Q}^{k-1} \oplus \mathfrak{d}^{*} \stackrel{\circ}{H}^{*} \mathfrak{Q}^{k+1} \oplus \mathfrak{H}^{k} .
$$


Here $\mathfrak{H}^{k}$ is the part of $L^{2} \mathbf{Q}^{k}$ perpendicular to the first two terms. We categorize the last term: Let $\mathfrak{c} \in \mathfrak{H}^{k}$, thus for all $\mathfrak{b} \in H \mathfrak{Q}^{k-1}$ and for all $\mathfrak{a} \in \mathfrak{d}^{*} \stackrel{\circ}{H}^{*} \mathfrak{Q}^{k+1}$

$$
(\mathfrak{c}, \mathfrak{d} \mathfrak{b})_{\mathfrak{F}}=0=\left(\mathfrak{c}, \mathfrak{d}^{*} \mathfrak{a}\right)_{\mathfrak{F}} .
$$

Then, by integration by parts, we have for all $\mathfrak{a}, \mathfrak{b}$ as above

$$
\left(\mathfrak{d}^{*} \mathfrak{c}, \mathfrak{b}\right)_{\mathfrak{F}}+\left(\operatorname{Tr} \mathfrak{b}, \operatorname{Tr}^{*} \mathfrak{c}\right)_{\partial Y}=0=(\mathfrak{d} \mathfrak{c}, \mathfrak{a})_{\mathfrak{F}} .
$$

Thus, $\mathfrak{S}^{k}$ has the definition stated in the theorem. That $\mathfrak{H}^{k}$ is isomorphic to cohomology follows directly from the decomposition since $\mathcal{N}\left(H \mathfrak{Q}^{k}, \mathfrak{D}\right)=\mathfrak{d} H \mathfrak{Q}^{k-1} \oplus \mathfrak{H}^{k}$.

Finally, it follows from Theorem 3.3 that $\mathfrak{S}^{k}$ is void if $Y$ is contractible.

Note that each cohomology class contains exactly one harmonic form, and that this minimizes the norm in its class (due to orthogonality). This parallels the classical situation where this property is used to prove Hodge's theorem, see, e.g., [19].

Lemma 3.11 For any $\mathfrak{a} \in L^{2} \mathfrak{Q}^{k}$, there exist $\mathfrak{b}_{\mathfrak{b}} \in H \mathfrak{Q}^{k-1}, \mathfrak{b}_{\mathfrak{b}^{*}} \in \stackrel{H}{H}^{*} \mathfrak{Q}^{k+1}$ and $\mathfrak{a}_{0} \in \mathfrak{H}^{k}$ such that

$$
\mathfrak{a}=\mathfrak{d} \mathfrak{b}_{\mathfrak{d}}+\mathfrak{d}^{*} \mathfrak{b}_{\mathfrak{b}}{ }^{*}+\mathfrak{a}_{0} .
$$

Proof Follows immediately from Theorems 3.4 and 3.3.

The role of boundary conditions on the spaces in Theorem 3.4 and Lemma 3.11 can be reversed, and for completeness we state:

Theorem 3.5 For any $\mathfrak{a} \in L^{2} \mathfrak{Q}^{k}$, there exist a Hodge decomposition $\mathfrak{a}_{\mathfrak{d}} \in \stackrel{\circ}{H} \mathfrak{Q}^{k}, \mathfrak{a}_{\mathfrak{d}^{*}} \in H^{*} \mathbf{Q}^{k}$ and $\mathfrak{a}_{0} \in \stackrel{\mathfrak{H}}{ }^{k}$ such that

$$
\mathfrak{a}=\mathfrak{a}_{\mathfrak{d}}+\mathfrak{a}_{\mathfrak{b}^{*}}+\mathfrak{a}_{0}
$$

while $\mathfrak{d}^{*} \mathfrak{a}_{\mathfrak{b}^{*}}=\mathfrak{d} \mathfrak{a}_{\mathfrak{d}}=\mathfrak{d}^{*} \mathfrak{a}_{0}=\mathfrak{d} \mathfrak{a}_{0}=0$. The space $\stackrel{\mathfrak{H}}{ }^{k}$ is referred to as the space of mixeddimensional harmonic forms with boundary conditions, and is defined by

$$
\stackrel{\circ}{\mathfrak{H}}^{k}=\left\{\mathfrak{c} \in \stackrel{\circ}{H}^{k} \cap H^{*} \mathfrak{Q}^{k} \mid \mathfrak{d}^{*} \mathfrak{c}=\mathfrak{d} \mathfrak{c}=0\right\} .
$$

The spaces $\stackrel{\mathfrak{H}}{ }^{k}$ are isomorphic to the cohomology of the de Rham complex $\left(\stackrel{H}{H} \mathfrak{Q}^{*}, \mathfrak{D}^{*}\right)$. The elements $\mathfrak{a}_{\mathfrak{d}}$ and $\mathfrak{a}_{\mathfrak{d}^{*}}$ can be represented as $\mathfrak{a}_{\mathfrak{d}}=\mathfrak{d} \mathfrak{b}_{\mathfrak{b}}$ and $\mathfrak{a}_{\mathfrak{b}^{*}}=\mathfrak{b}_{\mathfrak{b}^{*}}$ for $\mathfrak{b}_{\mathfrak{b}} \in \stackrel{\circ}{H} \mathfrak{Q}^{k-1}$, $\mathfrak{b}_{\mathfrak{b}^{*}} \in H \mathbf{Q}^{k+1}$.

Two subsequent corollaries follow from the above results.

Corollary 3.6 The cohomology of the sequence of mappings and spaces $\left(H^{*} \mathfrak{Q}^{\bullet}(\mathfrak{F}), \mathfrak{D}^{*}\right)$ has the same dimension as the cohomology of $\left(H^{*} \Lambda^{\bullet}(Y), \mathrm{d}^{*}\right)$.

Proof We note the equality of dimension of $\mathfrak{H}^{\bullet}$ and $\mathfrak{H}^{\bullet}$, as well as the cohomology spaces for $\left(H \mathfrak{Q}^{\bullet}(\mathfrak{F}), \mathfrak{d}\right),\left(H \Lambda^{\bullet}(Y), \mathrm{d}\right)$ and $\left(H^{*} \Lambda^{\bullet}(Y), \mathrm{d}^{*}\right)$.

Corollary 3.7 If $Y$ is a contractible domain, then for all $\mathfrak{a} \in H^{*} \mathfrak{Q}^{k}(\Omega)$ such that $\mathfrak{b}^{*} \mathfrak{a}=0$, there exists $\mathfrak{b} \in H^{*} \mathfrak{Q}^{k+1}$ such that $\mathfrak{\mathfrak { D }}^{*} \mathfrak{b}=\mathfrak{a}$. 
Proof Follows directly from the Hodge decomposition in Theorem 3.5 combined with the voidness of $\stackrel{\mathfrak{T}}{\mathrm{D}}^{k}$ for contractible domains from Corollary 3.6.

\subsection{Embeddings}

The mixed-dimensional spaces are related to each other by compact embeddings (abbreviated as Cᄃ). In particular, we will consider the space $\stackrel{\circ}{H} \mathfrak{Q}^{k} \cap H^{*} \mathbf{Q}^{k}$, and associate with it the norm

$$
\|\mathfrak{a}\|_{\mathfrak{D}, \mathfrak{b}^{*}}=\|\mathfrak{a}\|+\|\mathfrak{d} \mathfrak{a}\|+\left\|\mathfrak{b}^{*} \mathfrak{a}\right\|
$$

Theorem 3.6 The spaces $H \mathfrak{Q}^{k} \cap \stackrel{\circ}{H}^{*} \mathfrak{Q}^{k} \subset \subset L^{2} \mathfrak{Q}^{k}$ and $\stackrel{\circ}{H} \mathfrak{Q}^{k} \cap H^{*} \mathfrak{Q}^{k} \subset \subset L^{2} \mathfrak{Q}^{k}$.

Proof As shorthand, we let $G \mathfrak{Q}^{k}=\stackrel{\circ}{H} \mathfrak{Q}^{k} \cap H^{*} \mathfrak{Q}^{k}$, and likewise $G \Lambda^{k}=\stackrel{\circ}{H} \Lambda^{k} \cap H^{*} \Lambda^{k}$. We prove the theorem for this case, the other case being similar. We recall that for Lipschitz domains it holds that $G \Lambda^{k} \subset \subset L^{2} \Lambda^{k}[29]$.

It is clear from the definition of the inner products and norms that for all $\mathfrak{a} \in G \mathfrak{Q}^{k}$

$$
\|\mathfrak{a}\| \lesssim\|\mathfrak{a}\|_{\mathfrak{d}, \mathfrak{b}^{*}} \cdot
$$

It remains to show precompactness, which is to say that all bounded sequences $G \mathfrak{Q}^{k}$ have a subsequence that is convergent in $L^{2} \mathbf{Q}^{k}$.

Let $\mathfrak{a}_{m} \in G \boldsymbol{Q}^{k}$ be a bounded sequence. Since $G \boldsymbol{Q}^{k} \subset H \boldsymbol{Q}^{k}$, it follows from Lemma 3.10 that $l_{i} \mathfrak{a}_{m} \in H \Lambda^{k_{i}}\left(\Omega_{i}\right)$ for all nodes $i \in \mathfrak{F}$. Moreover, since $\left\|l_{i}\left(\mathfrak{b}^{*} \mathfrak{a}_{m}\right)\right\|_{\Omega_{i}}$ and $\left\|l_{i} \mathfrak{a}_{m}\right\|_{\Omega_{i}}$ are finite, it follows that $\left\|l_{i}\left(\mathrm{~d}^{*} \mathfrak{a}_{m}\right)\right\|_{\Omega_{i}}$ is finite as well. In turn, the functions $\boldsymbol{l}_{i} \mathfrak{a}_{m}$ lie in $H \Lambda^{k_{i}}\left(\Omega_{i}\right) \cap H^{*} \Lambda^{k_{i}}\left(\Omega_{i}\right)$. This allows us to construct a convergent subsequence, based on the following argument:

1. We argue by induction. For a given $d$, for all $i \in I$ and $j \in I_{i}^{d}$, let $l_{j} \mathfrak{a}_{m} \in G \Lambda^{k_{j}}\left(\Omega_{s_{j}}\right)$. Then, we can pass to a subsequence $\mathfrak{a}_{m^{\prime}}$ of $\mathfrak{a}_{m}$ such that $\iota_{j} \mathfrak{a}_{m^{\prime}}$ is convergent in $L^{2} \Lambda^{k_{j}}\left(\Omega_{s_{j}}\right)$. We denote by $\overline{\mathfrak{a}}_{m^{\prime}} \in G \mathbf{Q}^{k}$ any smooth and bounded extension of this limit sequence from Lemma 3.8. We now transform to a new sequence $\mathfrak{b}_{m}=\mathfrak{a}_{m^{\prime}}-\overline{\mathfrak{a}}_{m^{\prime}}$ which is still bounded.

2. If $\mathfrak{a}_{m}$ satisfies the premise in step 1 for some $d$, the sequence $\mathfrak{b}_{m}$ satisfies the premise for $d+1$ since clearly $l_{j} \mathfrak{b}_{m}=0$ for all $j \in I_{i}^{d}$, thus ${t_{j^{\prime}}} \mathfrak{b}_{m} \in G \Lambda^{k_{j^{\prime}}}\left(\Omega_{s_{j^{\prime}}}\right)$ for all $j^{\prime} \in I_{i}^{d+1}$.

3. For any sequence $\mathfrak{a}_{m}$, the premise in step 1 holds for $d=k_{i}$ since then $H \Lambda^{k_{i}}\left(\Omega_{s_{j}}\right)$ has no trace and $H \Lambda^{k_{i}}\left(\Omega_{s_{j}}\right)=\stackrel{\circ}{H} \Lambda^{k_{i}}\left(\Omega_{s_{j}}\right)$.

Thus, any bounded sequence in $G \mathbf{Q}^{k}$ has a subsequence that is convergent in $L^{2} \mathbf{Q}^{k}$, as desired.

\subsection{Poincaré-Friedrichs inequality}

We close this section by extending the Poincaré-Friedrichs inequality to the mixed-dimensional setting.

Theorem $\mathbf{3 . 7}$ (Poincaré-Friedrichs Inequality) For $\mathfrak{a} \in H \mathfrak{Q}^{k} \cap \stackrel{\circ}{H}^{*} \mathfrak{Q}^{k}$ or $\mathfrak{a} \in \stackrel{\circ}{H} \mathfrak{Q}^{k} \cap H^{*} \mathfrak{Q}^{k}$, with Hodge decompositions as in Sect. 3.5, it holds that 


$$
\|\mathfrak{a}\| \leq C\left(\left\|\mathfrak{d} \mathfrak{a}_{\mathfrak{b}^{*}}\right\|+\left\|\mathfrak{d}^{*} \mathfrak{a}_{\mathfrak{b}}\right\|\right)+\left\|\mathfrak{a}_{0}\right\| .
$$

Proof This follows directly from Theorem 3.6 and standard compactness arguments.

\section{Applications}

A large family of problems of physical interest arises as the minimization of suitably defined energies. This allows us to derive various problems of practical relevance, including variational problems and strong forms of differential equations. The requisite results from the fixed-dimensional elliptic differential equations carry over to the mixed-dimensional setting due to the results of Sect. 3; thus, we will in this section be brief in the exposition, and omit some generality and technical details. In particular, we will unless otherwise stated throughout this section only consider the case where $Y$ is contractible, the general case being similar. For a background on partial differential equations in the spirit of minimization problems and function spaces, especially on mixed form, please confer, i.e., [3, 7].

Section 4.2 then presents discrete subspaces of the mixed-dimensional spaces $H \mathbf{Q}^{k}$ and we show that these form discrete versions of the de Rham complex (3.41). To conclude the section, we show that $k=n$ leads to a model of flow in fractured porous media.

\subsection{Minimization problems}

We start by considering the minimization problem equivalent to a second-order elliptic differential equation involving the Hodge Laplacian for $\mathfrak{a} \in \stackrel{\circ}{H} \mathfrak{Q}^{k} \cap H^{*} \mathfrak{Q}^{k}$

$$
\mathfrak{a}=\underset{\mathfrak{a}^{\prime} \in H^{\prime} \mathfrak{Q}^{k} \cap H^{*} \mathfrak{Q}^{k}}{\arg \inf } J_{\mathfrak{r}}\left(\mathfrak{a}^{\prime}\right),
$$

where we define the functional by

$$
J_{\mathfrak{r}, \mathfrak{f}}\left(\mathfrak{a}^{\prime}\right)=\frac{1}{2}\left(\mathfrak{x} \mathfrak{D}^{*} \mathfrak{a}^{\prime}, \mathfrak{d}^{*} \mathfrak{a}^{\prime}\right)+\frac{1}{2}\left(\mathfrak{r}^{*} \mathfrak{d} \mathfrak{a}^{\prime}, \mathfrak{d} \mathfrak{a}^{\prime}\right)-\left(\mathfrak{f}, \mathfrak{a}^{\prime}\right) .
$$

Coefficients will be termed symmetric positive definite if a scalar $r$ exists such that

$$
\begin{aligned}
& \inf _{\mathfrak{b} \in \mathfrak{d} H \mathfrak{Q}^{k}}(\mathfrak{x} \mathfrak{b}, \mathfrak{b}) \geq r>0 \quad \text { and } \quad(\mathfrak{x} \mathfrak{a}, \mathfrak{b})=(\mathfrak{a}, \mathfrak{r} \mathfrak{b}) \quad \text { for all } \mathfrak{a}, \mathfrak{b} \in H \mathfrak{Q}^{k} . \\
& \|\mathfrak{b}\|_{\Omega}=1
\end{aligned}
$$

Theorem 4.1 For contractible domains $Y$, the functional $J_{\mathfrak{r}, \mathfrak{f}}$ has a unique minimum in $\stackrel{\circ}{H} \mathfrak{Q}^{k} \cap H^{*} \mathfrak{Q}^{k}$ for symmetric positive definite coefficients $\mathfrak{x}$ and $\mathfrak{r}^{*}$.

Proof The unique solvability of (4.1) is equivalent to the coercivity of the quadratic term [11]. With respect to the norm (3.54), we obtain coercivity by the Poincaré-Friedrichs inequality (subject to the conditions in the proof):

$$
\frac{1}{2}\left(\mathfrak{x} \mathfrak{b}^{*} \mathfrak{a}, \mathfrak{b}^{*} \mathfrak{a}\right)+\frac{1}{2}\left(\mathfrak{r}^{*} \mathfrak{d} \mathfrak{a}, \mathfrak{d} \mathfrak{a}\right) \geq \min \left(r, r^{*}\right)\left(\|\mathfrak{d} \mathfrak{a}\|+\left\|\mathfrak{D}^{*} \mathfrak{a}\right\|\right) \gtrsim\|\mathfrak{a}\|_{\mathfrak{d}, \mathfrak{b}^{*}}
$$


From calculus of variations, we know that the minimum of (4.1) satisfies the Euler-Lagrange equations when $J_{\mathfrak{r}, \mathfrak{f}}$ is differentiable. Thus, $\mathfrak{a} \in \stackrel{\circ}{H} \mathbf{Q}^{k} \cap H^{*} \mathbf{Q}^{k}$ satisfies

$$
\left(\mathfrak{r} \mathfrak{d}^{*} \mathfrak{a}, \mathfrak{b}^{*} \mathfrak{a}^{\prime}\right)+\left(\mathfrak{r}^{*} \mathfrak{d} \mathfrak{a}, \mathfrak{d} \mathfrak{a}^{\prime}\right)=\left(\mathfrak{f}, \mathfrak{a}^{\prime}\right) \quad \text { for all } \mathfrak{a}^{\prime} \in \stackrel{\circ}{H} \mathfrak{Q}^{k} \cap H^{*} \mathfrak{Q}^{k} .
$$

The existence of solutions of (4.5) follows directly from Theorem 4.1. Uniqueness follows from the coercivity of the bilinear form $\left(\mathfrak{x} \mathfrak{b}^{*} \mathfrak{a}, \mathfrak{d}^{*} \mathfrak{a}^{\prime}\right)+\left(\mathfrak{r}^{*} \mathfrak{d} \mathfrak{a}, \mathfrak{d} \mathfrak{a}^{\prime}\right)$.

Remark 4.1 It is important to note the structure of the coefficient $\mathfrak{r}$ and $\mathfrak{r}^{*}$. Due to the definition of the inner product (3.6) and function spaces on the forest $\mathfrak{F}$, these coefficients operate both on function values on manifolds $\Omega_{i}$, as well as on the traces of functions, i.e., function values of the whole forest $\mathfrak{F}$ (but not the co-traces). These aspects extend the concept of a "material property", and form useful guidance in the design of constitutive laws for mixed-dimensional models.

Remark 4.2 In some applications, it will not be appropriate to include a differential equation at all intersections, and rather only consider jump conditions. This is reflected in a degeneracy of the coefficient for that sub-manifold. Thus, it is of interest to consider the case where $l_{i} \mathfrak{x} \rightarrow 0$ on some manifolds. This is outside the scope of the current paper, but has been considered in related work [9].

\subsubsection{Mixed formulation}

In order to capture explicitly physical quantities and properties of a problem, it is frequently preferable to consider a so-called mixed problem obtained by setting $\mathfrak{b}=-\mathfrak{r} \mathfrak{d}^{*} \mathfrak{a}$. Thus, we consider: Find $(\mathfrak{a}, \mathfrak{b}) \in H \mathfrak{Q}^{k} \times H \mathfrak{Q}^{k-1}$ which satisfy

$$
\begin{aligned}
\left(\mathfrak{r}^{-1} \mathfrak{b}, \mathfrak{b}^{\prime}\right)+\left(\mathfrak{a}, \mathfrak{d} \mathfrak{b}^{\prime}\right) & =0 \quad \text { for all } \mathfrak{b}^{\prime} \in \mathfrak{Q}^{k-1}, \\
\left(\mathfrak{d} \mathfrak{b}, \mathfrak{a}^{\prime}\right)+\left(\mathfrak{r}^{*} \mathfrak{d a}, \mathfrak{d} \mathfrak{a}^{\prime}\right) & =\left(\mathfrak{f}, \mathfrak{a}^{\prime}\right) \quad \text { for all } \mathfrak{a}^{\prime} \in \mathfrak{Q}^{k}
\end{aligned}
$$

Equations (4.6) have the practical advantage that they do not require the coderivative. In applications, the exterior derivative often encompasses a conservation principle [3, 18, 22], and it is therefore also advantageous in applied computations to represent it explicitly (for an applied perspective, see also discussions in, e.g., [14, 20, 26, 31]).

Theorem 4.2 For uniformly bounded and non-degenerate coefficients $\mathfrak{r}$ and $\mathfrak{r}^{*}$, problem (4.6) is well-posed.

Proof The well-posedness of the mixed formulation follows due to the inf-sup condition on the coupling term $[5,7]$. Due to the Hodge decomposition and Poincare inequality this holds by a similar calculation as in the fixed-dimensional case, see, e.g., the proof of Theorem 7.2 in [3].

\subsubsection{Strong formulation}

We will only consider the strong form of the mixed-dimensional PDE from a formal perspective. From a practical viewpoint, the strong form of the equations is most useful in applications, as they explicitly give the engineer and practitioner guidelines for the interpretation of 
the parameter functions and the physical and constitutive laws. We thus briefly state and discuss the strong form of the equations here.

For sufficiently regular $\mathfrak{a} \in \mathfrak{\Omega}^{k}(\Omega)$ :

$$
\mathfrak{d}\left(\mathfrak{x} \mathfrak{d}^{*} \mathfrak{a}\right)+\mathfrak{d}^{*}\left(\mathfrak{r}^{*} \mathfrak{d} \mathfrak{a}\right)=\mathfrak{f} .
$$

Due to the availability of Helmholtz (and Hodge) decompositions, the mixed-dimensional Hodge-Laplacian inherits the structure of the fixed-dimensional case, and thus, Eq. (4.7) can be split into separate sub-problems. Indeed, for a Hodge decomposition (Theorem 3.4) of the solution and the right-hand side, $\mathfrak{a}=\mathfrak{a}_{\mathfrak{b}^{*}}+\mathfrak{a}_{\mathfrak{b}}+\mathfrak{a}_{0}$ and $\mathfrak{f}=\mathfrak{f}_{\mathfrak{b}^{*}}+\mathfrak{f}_{\mathfrak{b}}+\mathfrak{f}_{0}$ the Hodge Laplacian separates, and it holds that

$$
\begin{gathered}
\mathfrak{a}_{0}=\mathfrak{f}_{0}, \\
\mathfrak{d}\left(\mathfrak{x} \mathfrak{d}^{*} \mathfrak{a}_{\mathfrak{d}}\right)=\mathfrak{f}_{\mathfrak{d}}, \\
\mathfrak{d}^{*}\left(\mathfrak{r}^{*} \mathfrak{d} \mathfrak{a}_{\mathfrak{d}^{*}}\right)=\mathfrak{f}_{\mathfrak{d}^{*}} .
\end{gathered}
$$

Equations (4.8a)-(4.8c) have unique solutions in the subspaces where the appropriate differentials are zero, as per the decomposition. Thus, we see that the Hodge Laplacian encompasses the traditional Laplacian equations, also in the mixed-dimensional setting. When $Y$ is contractible, Eq. (4.8a) is of course irrelevant.

\subsection{Discrete subspaces}

Finite-dimensional approximations of Eqs. (4.5) and (4.6) can be constructed based on finite element exterior calculus [3]. For that purpose, this section presents two families of finite element spaces that form discrete versions of the mixed-dimensional de Rham complex.

Let $X_{h, i}$ be a simplicial tessellation of $X_{i}$. Then, following [3], let $P_{r} \Lambda^{k}\left(X_{h, i}\right)$ denote the conforming finite element space on $X_{h, i}$ containing all polynomial $k$-forms up to degree $r$ and let $P_{r}^{-} \Lambda^{k}\left(X_{h, i}\right)$ be the reduced family. Following the same product structure as (3.5), we define

$$
P_{r}^{-} \mathfrak{Q}^{k}(\mathfrak{F}):=\prod_{i \in I} P_{r}^{-} \Lambda^{k_{i}}\left(X_{h, i}\right), \quad P_{r} \mathfrak{Q}^{k}(\mathfrak{F}):=\prod_{i \in I} P_{r+\left(n-d_{i}\right)} \Lambda^{k_{i}}\left(X_{h, i}\right) .
$$

Here, we recall that $k_{i}:=k-\left(n-d_{i}\right)$. We note that these finite element spaces are conforming in the sense that $P_{r}^{-} \mathfrak{Q}^{k}(\mathfrak{F}) \subseteq P_{r} \mathfrak{Q}^{k}(\mathfrak{F}) \subseteq H \mathfrak{Q}^{k}(\mathfrak{F})$.

It is easy to check that $\mathbb{d} P_{r}^{-} \mathbf{2}^{k} \subseteq P_{r}^{-} \mathbf{2}^{k+1}$ and $\mathbb{d} P_{r} \mathbf{Q}^{k} \subseteq P_{r-1} \mathbf{2}^{k+1}$. Combining this with the discrete complexes presented in [3], we obtain

$$
P_{r}^{-} \mathfrak{Q}^{k} \stackrel{\mathfrak{d}}{\rightarrow} P_{r}^{-} \mathfrak{Q}^{k+1}, \quad P_{r} \mathfrak{Q}^{k} \stackrel{\mathfrak{d}}{\rightarrow} P_{r-1} \mathfrak{Q}^{k+1} .
$$

In turn, $\left(P_{r}^{-} \mathfrak{Q} \cdot \mathfrak{D}\right)$ and $\left(P_{r-.} \mathfrak{L} \cdot \mathfrak{D}\right)$ form discrete cochain complexes.

To make this example more concrete, let us consider $n=3$. We adhere to the following convention for differential operators. The 3D de Rham complex has the operators gradient $\nabla$, curl $\nabla \times$, and divergence $\nabla \cdot$. In $2 \mathrm{D}$, we represent the de Rham complex as rotated gradient $\nabla \times=-n \times \nabla$ (where $n$ is normal to the manifold) and divergence $\nabla \cdot$. In $1 \mathrm{D}$, the differential is $d_{x}$, the spatial derivative. Additionally, we point out that the trace of functions $a_{i}$ in $H \Lambda^{d_{i}-1}\left(X_{i}\right)$ and $H \Lambda^{d_{i}-2}\left(X_{i}\right)$ is given with respect to the outer normal vector $n$ as $a_{i} \cdot n$ and 
$a_{i} \times n$, respectively. We can now decompose the reduced spaces of lowest order to form the following diagram:
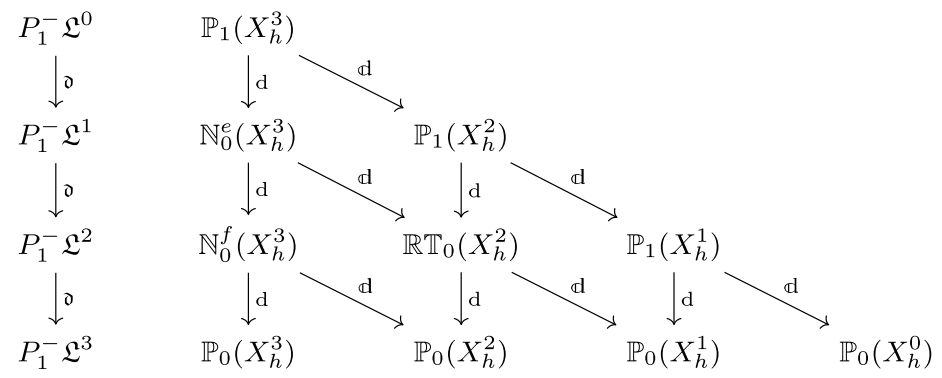

Here, we used the notation $X_{h}^{d}:=\bigcup_{i \in I^{d}} X_{h, i}$. The local finite element spaces are given by the following: $\mathbb{P}_{1}$ denotes the space of linear Lagrange elements, $\mathbb{P}_{0}$ denotes the piecewise constants, $\mathbb{R T}_{0}$ is the space of Raviart-Thomas elements of lowest order, and the spaces $\mathbb{N}_{0}^{f}$ and $\mathbb{N}_{0}^{e}$ refer to the Nédélec elements of the first kind of lowest order with degrees of freedom on faces and edges, respectively.

Remark 4.3 Diagram (4.11) is equivalent to the complex presented in [21] if each $X_{i}=X_{h, i}$ is a simplex. In turn, that work considers a special case of the proposed framework concerning discrete distributional differential forms on simplicial complexes.

On the other hand, the family $P_{r} \mathbf{2}^{k}$ contains the Nédélec elements of the second kind in $3 \mathrm{D}$, the two-dimensional Brezzi-Douglas-Marini elements, and the continuous and discontinuous Lagrange elements. A decomposition diagram of this family in the style of (4.11) is presented in [8, Sec. 4.1.2].

These finite element spaces can directly be applied for problems of the form (4.5) and (4.6). In particular, for $k=n$, this leads to a finite-dimensional approximation that has the expected approximation properties based on preliminary computational examples [27]. While not covered by the present analysis, finite-dimensional approximations of the mixed variational problems can also be shown to allow for degeneracies in the coefficients which are of importance in practical computations [9].

\subsection{Flow in fractured porous media}

In terms of classical notation, expressions such as (4.8) become very tedious when expressed for general manifolds. We therefore focus on the example geometry of Fig. 1 in this section and show how the interpretation of (4.8) for $k=n=2$ is related to some existing models in literature concerning modeling of porous media. Throughout the section we will assume sufficient regularity to express equations in strong form, and not detail function spaces.

From Eq. (3.3), we note that the case of $k=2$ concerns scalar variables associated with all submanifolds, i.e., $\mathfrak{a} \in \mathfrak{Q}^{2}(\Omega)$ is decomposed into $a_{i} \in \Lambda^{d_{i}}\left(\Omega_{i}\right)$ for all subdomains. Similarly, the coefficients $\mathfrak{r}$ are denoted by $r_{i}$ on all roots $i \in I$ and by $r_{j}$ with $j \in I_{i}^{d_{i}-1}$ for all components associated with the boundaries of subdomains (see also remark 4.1). 
Equations (4.8a) and (4.8c) are void since the domain $Y$ is contractible and there is no $\mathfrak{Q}^{n+1}$; thus, only Eq. (4.8b) is of relevance. From the definitions of the mixed-dimensional exterior derivative and coderivative, we obtain the following system of equations, where we first state the differential equations on (sub)manifolds:

$$
\begin{gathered}
\nabla \cdot\left(-r_{4} \nabla a_{4}\right)=f_{4} \quad \text { on } \Omega_{4}, \\
d_{x} \cdot\left(-r_{3} d_{x} a_{3}\right)+\sum_{j \in \gamma_{3}^{-1}}\left(-r_{j}\left(a_{4}-a_{3}\right)\right)=f_{3} \quad \text { on } \Omega_{3}, \\
\sum_{j \in \gamma_{2}^{-1}}\left(-r_{j}\left(a_{3}-a_{2}\right)\right)=f_{2} \quad \text { on } \Omega_{2}, \\
\sum_{j \in \gamma_{1}^{-1}}\left(-r_{j}\left(a_{3}-a_{1}\right)\right)=f_{1} \quad \text { on } \Omega_{1} .
\end{gathered}
$$

The index sets in this example are given by $\gamma_{3}^{-1}:=\left\{j \in \mathfrak{S}_{4} \mid s_{j}=3\right\}$, $\gamma_{2}^{-1}:=\left\{j \in \mathfrak{S}_{3} \mid s_{j}=2\right\}$, and $\gamma_{1}^{-1}:=\left\{j \in \mathfrak{S}_{3} \mid s_{j}=1\right\}$.

These are coupled by continuity conditions for continuous and discrete evaluation of normal components of vectors $\left(n_{j}\right.$ is the normal vector of $\partial_{j} \Omega_{\hat{\jmath}}$ oriented outward with respect to $\Omega_{\hat{J}}$ :

$$
\begin{aligned}
& n_{j} \cdot\left(-r_{4} \nabla a_{4}\right)-r_{j}\left(a_{4}-a_{s_{j}}\right)=0 \quad \text { at } \partial_{j} \Omega_{4} \text { and for all } j \in I_{4}^{1}, \\
& n_{j} \cdot\left(-r_{3} d_{x} a_{3}\right)-r_{j}\left(a_{3}-a_{s_{j}}\right)=0 \quad \text { at } \partial_{j} \Omega_{3} \text { and for all } j \in I_{3}^{0} .
\end{aligned}
$$

These equations correspond to an elliptic PDE on each manifold, coupled together with jump conditions from the higher-dimensional manifolds. From the perspective of a scalar conservation law, the jump conditions ensure that mass (or energy) is conserved. These equations are therefore truly mixed-dimensional, in that Eq. (4.12) are not only posed on submanifolds of different dimensions, but are explicitly coupled.

From a physical perspective, we note that Eq. (4.12) correspond to, e.g., Darcy flow in a fractured porous medium. In this sense, Eqs. (4.12a) and (4.12b) represent flows in the bulk and the fracture, respectively. The balance Eqs. (4.12c) and (4.12d) ensure mass conservation at fracture ends and, generally, intersections. The continuity conditions are then continuity of normal flux from higher to lower-dimensional domains. Such models have been widely adopted and studied over the last decade (see, e.g., [2, 16, 17]).

We emphasize the interpretation of the coefficients appearing in (4.12). For each domain $\Omega_{i}$, the coefficient $r_{i}$ has the interpretation of permeability of flow in the directions within the domain, that is to say parallel to the bulk and fractures. In contrast, the coefficients $r_{j}$ (with $j$ not a root) are in a sense the permeability to flow across the internal boundaries, which is physically associated with the permeability across the neighboring subdomains. As a small remark, we note that the coefficients $r_{i}$ of a geological medium will in general be anisotropic tensors of rank $d_{i}$. If we follow the interpretation given above, this implies that for $d_{i}<n$ the coefficients $r_{i}$ together with the coefficients $r_{j}$ of its neighbors can be jointly seen as tensors of rank $n$-however, with the restriction that the main directions of the anisotropy are aligned normal and parallel to the subdomain $\Omega_{i}$. 
Considering the limit of $r_{i}, f_{i} \rightarrow 0$ for $i \in I$ with $d_{i}<n$, these equations have also been adopted in order to study geological features such as faults or aquitards (for examples where these equations have been applied, see, e.g., [4, 28]). In this limit, the differential terms in Eq. (4.12b) are omitted, and $a_{i}$ can be eliminated algebraically from the equations for $d_{i}<n$.

A degenerate form of Eqs. (4.12a)-(4.12f) also appears in domain decomposition algorithms, in the further limit of $r_{j} \rightarrow \infty$, for all $j \notin I$. In this limit, only Eq. (4.12a) remains as a differential equation, while Eqs. (4.12b)-(4.12f) reduce to continuity conditions in the Neumann and Dirichlet sense [33].

Equation (4.12) are the standard way of expressing the equations for flow in fractured porous media. It is clear from their repetitive structure that a unified hierarchical mixeddimensional formulation, such as given in Eq. (4.8b), provides a much simpler representation of the structure in the equations. This simplification facilitates the theoretical analysis of (4.12) using the results from Sects. 3 and 4.

\section{Conclusions}

In this manuscript we introduce a semi-discrete exterior differential operator, in such a way that it corresponds to hierarchically coupled mixed-dimensional partial differential equations. We show that this mixed-dimensional exterior derivative inherits standard properties from fixed-dimensional calculus, including a codifferential, Hodge decomposition, Poincare's lemma and inequality, and Stokes' theorem. Our approach leads to mixed-dimensional structures of $k$-forms, similar to those of fixed-dimensional calculus; however, the familiar Hodge duality is not available, and there is no wedge product.

Within this setting, we define mixed-dimensional Hodge Laplacians, and show that they correspond to well-posed minimization problems. We identify equations familiar from applications in fractured porous media. As such, the present analysis provides a unified approach for handling problems in continuum mechanics wherein inclusions with high aspect ratios are modeled as lower-dimensional.

Acknowledgements Open access funding provided by Royal Institute of Technology. The authors wish to thank Snorre Christiansen, Bernd Flemisch, Gunnar Fløystad, Alessio Fumagalli, Eirik Keilegavlen, Olivier Verdier, Ivan Yotov, and Ludmil Zikatanov for valuable comments and discussions on this topic.

Funding This research was supported in part by the Norwegian Research Council Grants: 233736, 250223.

Open Access This article is licensed under a Creative Commons Attribution 4.0 International License, which permits use, sharing, adaptation, distribution and reproduction in any medium or format, as long as you give appropriate credit to the original author(s) and the source, provide a link to the Creative Commons licence, and indicate if changes were made. The images or other third party material in this article are included in the article's Creative Commons licence, unless indicated otherwise in a credit line to the material. If material is not included in the article's Creative Commons licence and your intended use is not permitted by statutory regulation or exceeds the permitted use, you will need to obtain permission directly from the copyright holder. To view a copy of this licence, visit http://creativecommons.org/licenses/by/4.0/. 


\section{Appendix}

\section{Proof of Theorem 3.3}

Proof From Lemmas A.1-A.3, it follows that without loss of generality, it is sufficient to consider functions $\mathfrak{a} \in H \mathbf{Q}^{k}$ where the only non-zero component is on $\Omega^{n}$. Consider therefore a form $\mathfrak{a} \in H \mathfrak{Q}^{k}$ with $\mathfrak{d} \mathfrak{a}=0$ and $a_{i}=0$ for all $i \in \mathfrak{F}$ with $d_{i}<n$. It follows that $l_{i}(\mathbb{d} \mathfrak{a})=0$ for $i \in I^{n-1}$. In turn, the internal boundaries have no contribution on computations of integrals, which therefore depend only on the geometry of $Y$ itself. Since cohomology can be computed using integrals by de Rham's theorem, the claim follows.

Lemma A.1 For each $\mathfrak{a} \in H \mathfrak{Q}^{n}$, there exists $a \mathfrak{b} \in H \mathfrak{Q}^{n-1}$ such that $\iota_{i}(\mathfrak{a}-\mathfrak{d b})=0$ on all DAGs $\mathfrak{\Im}_{i}$ with $d_{i}<n$.

Proof Take $\mathfrak{a} \in H \mathfrak{Q}^{n}$ as given and proceed according to the following three steps.

Step $A$. We start by considering $i \in I^{0}$. For any such $i$, we can construct a function $\mathfrak{c} \in H \mathfrak{Q}^{n-1}$ using the extension operators from Lemma 3.8, such that

(a) $l_{i}(\mathbb{d} \mathfrak{c})=a_{i}$

(b) $l_{j}(\mathbb{d} \mathfrak{c})=0$ for all $j \in I^{0} \backslash\{i\}$

(c) $c_{j^{\prime}}=0$ for $j^{\prime} \in I$ if $d_{j^{\prime}} \neq 1$ or if $I_{j^{\prime}} \cap \gamma_{i}^{-1}=\emptyset$.

We define the corrected function

$$
\mathfrak{a}^{\prime}=\mathfrak{a}-\mathfrak{d} \mathfrak{c} .
$$

Note that $\mathfrak{a}^{\prime}$ is in the same cohomology class as $\mathfrak{a}$ with $a_{i}^{\prime}=0$, and we therefore rename $\mathfrak{a}^{\prime}$ as $\mathfrak{a}$.

Step B. We repeat Step A for all $i \in I^{0}$.

Step $C$. Steps A and B are repeated for all $i \in I$ with $0<d_{i}<n$ in increasing order of dimension. For each $i$, the correction function $c \in H \mathfrak{Q}^{n-1}$ then satisfies

(a) $l_{i}(\mathbb{d} \mathfrak{c})=a_{i}$

(b) $l_{j}(\mathbb{d} \mathfrak{c})=0$ for all $j \in I^{d_{i}} \backslash\{i\}$

(c) $c_{j^{\prime}}=0$ for $j^{\prime} \in I$ if $d_{j^{\prime}} \neq d_{i}+1$ or if $I_{j^{\prime}} \cap \gamma_{i}^{-1}=\emptyset$.

Defining $\mathfrak{b}$ as the sum of all consecutive correction functions, we arrive at the claim.

Lemma A.2 Given $n \leq 3$, for each $\mathfrak{a} \in H \mathfrak{Q}^{n-1}$ with $\mathfrak{d} \mathfrak{a}=0$, there exists $a \mathfrak{b} \in H \mathfrak{Q}^{n-2}$ such that $\iota_{i}(\mathfrak{a}-\mathfrak{d} \mathfrak{b})=0$ on all DAGs $\mathfrak{S}_{i}$ with $d_{i}<n$.

Proof Take $\mathfrak{a} \in H \mathfrak{\Omega}^{n-1}$ as given, then by definition $a_{i}=0$ for all $i \in I^{0}$. We nonetheless first introduce a correction according to each zero-dimensional manifold such that the corresponding traces of $\mathfrak{a}$ vanish. We continue according to the following steps.

Step A. Consider any fixed $i \in I^{0}$. By continuity, each $a_{j}$ with $j \in \gamma_{i}^{-1}$ represents a neighboring trace of $\mathfrak{a}$ from the 1-manifolds adjacent to $i$. We then introduce $\mathfrak{c} \in H \mathbf{Q}^{n-2}$, using the extension operators from Lemma 3.8, such that

(a) $l_{j}(\mathbb{d} \mathfrak{c})=a_{j}$ for each $j \in \gamma_{i}^{-1}$

(b) $l_{j^{\prime}}(\mathbb{d} \mathfrak{c})=0$ for all $j^{\prime} \in I_{l}^{0} \backslash \gamma_{i}^{-1}$ with $l \in I^{1}$ 
(c) $c_{j^{\prime \prime}}=0$ for $j^{\prime \prime} \in I$ if $d_{j^{\prime \prime}} \neq 2$ or if $I_{j^{\prime \prime}} \cap \gamma_{i}^{-2}=\emptyset$.

Constraint (a) above involves solving a system of $\left|\gamma_{i}^{-1}\right|$ equations with $\left|\gamma_{i}^{-2}\right|=\left|\bigcup_{j \in \gamma_{i}^{-1}} \gamma_{j}^{-1}\right|$ unknowns. This system is solvable by the fact that $l_{i}(\mathbb{d} \mathfrak{a})=0$ and the assumed geometry.

We define the corrected function $\mathfrak{a}^{\prime}=\mathfrak{a}-\mathfrak{d} \mathfrak{c}$. Note that $\mathfrak{a}^{\prime}$ is in the same cohomology class as $\mathfrak{a}$ with $a_{j}^{\prime}=0$, and we therefore rename $\mathfrak{a}^{\prime}$ as $\mathfrak{a}$.

Step B. We repeat Step A for all $i \in I^{0}$.

Step $C$. Consider any fixed $i \in I^{1}$. We construct a function $c \in H \mathfrak{Q}^{n-2}$ such that

(a) $l_{i}(\mathbb{d} \mathfrak{c})=a_{i}$

(b) $l_{j}(\mathbb{d} \mathfrak{c})=0$ for all $j \in I^{1} \backslash\{i\}$

(c) $c_{j}=0$ for $j \in I$ if $d_{j} \neq 2$ or if $I_{j} \cap \gamma_{i}^{-1}=\emptyset$.

Introducing $\mathfrak{a}^{\prime}=\mathfrak{a}-\mathfrak{b} \mathfrak{c}$, we note that $a_{i}^{\prime}=0$ and we rename $\mathfrak{a}^{\prime}$ as $\mathfrak{a}$.

Step D. Step C is repeated for all $i \in I^{1}$. The result is now shown for $n=2$.

Step E. Continuing with $n=3$, consider any fixed $i \in I^{2}$. We construct a function $\mathfrak{c} \in H \mathbf{Q}^{n-2}$, using the extension operators from Lemma 3.8, such that

(a) $l_{i}(\mathbb{d} \mathfrak{c})=a_{i}$

(b) $l_{j}(\mathbb{d} \mathfrak{c})=0$ for all $j \in I^{2} \backslash\{i\}$

(c) $c_{j}=0$ for $j \in I$ if $d_{j} \neq 3$ or if $I_{j} \cap \gamma_{i}^{-1}=\emptyset$.

Introducing $\mathfrak{a}^{\prime}=\mathfrak{a}-\mathfrak{d} \mathfrak{c}$, we note that $a_{i}^{\prime}=0$ and we rename $\mathfrak{a}^{\prime}$ as $\mathfrak{a}$.

Step $F$. Repeating Step E for all $i \in I^{2}$ proves the result for $n=3$.

Lemma A.3 Given $n=3$, for each $\mathfrak{a} \in H \mathfrak{Q}^{1}$ with $\mathfrak{d} \mathfrak{a}=0$, there exists $a \mathfrak{b} \in H \mathfrak{Q}^{0}$ such that $l_{i}(\mathfrak{a}-\mathfrak{d} \mathfrak{b})=0$ on all DAGs $\mathfrak{S}_{i}$ with $d_{i}<n$.

Proof As in the previous lemmas, we correct a given form $\mathfrak{a}$. Note that here, we only need to correct $\mathfrak{a}$ on DAGs $\mathfrak{S}_{i}$ with $d_{i}=2$. We start with the zero-dimensional nodes in these DAGs and progress in increasing order of dimension.

Step A. Consider $i \in I^{0}$ fixed. We construct $c \in H \mathbf{Q}^{0}$ such that

(a) $l_{j}(\mathbb{d} \mathfrak{c})=a_{j}$ for each $j \in \gamma_{i}^{-2}$

(b) $l_{j^{\prime}}(\mathbb{d} \mathfrak{c})=0$ for all $j^{\prime} \in I_{l}^{0} \backslash \gamma_{i}^{-2}$ with $l \in I^{2}$

(c) $c_{j^{\prime \prime}}=0$ for $j^{\prime \prime} \in I$ if $I_{j^{\prime \prime}} \cap \gamma_{i}^{-3}=\emptyset$.

In the above, (a) involves solving a system of $\left|\gamma_{i}^{-2}\right|$ equations with $\left|\gamma_{i}^{-3}\right|=\left|\bigcup_{j \in \gamma_{i}^{-2}} \gamma_{j}^{-1}\right|$ unknowns. Due to the fact that $l_{j^{\prime}}(\mathbb{d} \mathfrak{a})=0$ for all $j^{\prime} \in \gamma_{i}^{-1}$ this system reduces to $\left|\gamma_{i}^{-2}\right|-\left|\gamma_{i}^{-1}\right|$ equations, which is solvable by the geometric constraints.

We define the corrected function $\mathfrak{a}^{\prime}=\mathfrak{a}-\mathfrak{d} \mathfrak{c}$. Note that $\mathfrak{a}^{\prime}$ is in the same cohomology class as $\mathfrak{a}$ with $a_{j}^{\prime}=0$, and we therefore rename $\mathfrak{a}^{\prime}$ as $\mathfrak{a}$.

Step B. Repeat step A for all $i \in I^{0}$.

Step $C$. Consider $i \in I^{1}$ fixed. Let $j \in \gamma_{i}^{-1}$ and consider $a_{j}$. We construct $c \in H \mathbf{Q}^{0}$ such that

(a) $l_{j}(\mathbb{d} \mathfrak{c})=a_{j}$ for each $j \in \gamma_{i}^{-1}$

(b) $l_{j^{\prime}}(\mathbb{d} \mathfrak{c})=0$ for all $j^{\prime} \in I_{l}^{1} \backslash \gamma_{i}^{-1}$ with $l \in I^{2}$ 
(c) $c_{j^{\prime \prime}}=0$ for $j^{\prime \prime} \in I$ if $I_{j^{\prime \prime}} \cap \gamma_{i}^{-2}=\emptyset$.

In the above, (a) involves solving a system of $\left|\gamma_{i}^{-1}\right|$ equations with $\left|\gamma_{i}^{-2}\right|$ unknowns. Due to the fact that $l_{i}(\mathbb{d} \mathfrak{a})=0$ this system is solvable. Introducing $\mathfrak{a}^{\prime}=\mathfrak{a}-\mathfrak{b} \mathfrak{c}$, we note that $a_{j}^{\prime}=0$ and we rename $\mathfrak{a}^{\prime}$ as $\mathfrak{a}$.

Step D. Repeat step C for all $i \in I^{1}$.

Step E. Consider $i \in I^{2}$ fixed. We construct $c \in H \mathfrak{Q}^{0}$ such that

(a) $l_{i}(\mathbb{d} \mathfrak{c})=a_{i}$

(b) $l_{j}(\mathbb{d} \mathfrak{c})=0$ for all $j \in I^{2} \backslash\{i\}$

(c) $c_{j^{\prime}}=0$ for $j \in I$ if $I_{j} \cap \gamma_{i}^{-1}=\emptyset$.

Introducing $\mathfrak{a}^{\prime}=\mathfrak{a}-\mathfrak{d} \mathfrak{c}$, we note that $a_{i}^{\prime}=0$ and we rename $\mathfrak{a}^{\prime}$ as $\mathfrak{a}$.

Step F. Repeat step E for all $i \in I^{2}$ to obtain the result.

\section{References}

1. Adams, R., Fournier, J.: Sobolev Spaces. Elsevier Science, Pure and Applied Mathematics (2003)

2. Alboin, C., Jaffré, J., Roberts, J.E., Serres, C.: Domain decomposition for flow in porous media with fractures. In: 14th Conference on Domain Decomposition Methods in Sciences and Engineering (1999)

3. Arnold, D.N., Falk, R.S., Winther, R.: Finite element exterior calculus, homological techniques, and applications. Acta Numerica 15, 1-155 (2006)

4. Avci, C.B.: Evaluation of flow leakage through abandoned wells and boreholes. Water Resour. Res. 30(9), 2565-2578 (1994)

5. Babuska, I., Aziz, A.K.: Survey lectures on the mathematical foundations. In: The Mathematical Foundations of the Finite Element Method with Applications to Partial Differential Equations, Academic Press, New York, pp. 1-359 (1972)

6. Bear, J.: Hydraulics of Groundwater. McGraw-Hill, New York (1979)

7. Boffi, D., Brezzi, F., Fortin, M.: Mixed Finite Elements and Applications. Springer, Heidelberg (2013)

8. Boon, W.M.: Conforming Discretizations of Mixed-Dimensional Partial Differential Equations. Ph.D. Thesis, The University of Bergen (2018)

9. Boon, W.M., Nordbotten, J.M., Yotov, I.: Robust discretization of flow in fractured porous media. SIAM J. Numer. Anal. 56(4), 2203-2233 (2018)

10. Bott, R., Tu, L.W.: Differential Forms in Algebraic Topology. Springer, New York (1982)

11. Braess, D.: Finite Elements: Theory. Fast Solvers and Applications in Solid Mechanics. Cambridge University Press, Cambridge (2007)

12. Buffa, A., Costabel, M., Sheen, D.: On traces for $\mathrm{H}(\mathrm{curl}, \Omega)$ in Lipschitz domains. J. Math. Anal. Appl 276(2), 845-867 (2002)

13. Buffa, A.P., Ciarlet, J.: On traces for functional spaces related to Maxwell's equations. Part 1: an integration by parts formula in Lipschitz polyhedra. Math. Methods Appl. Sci. 24, 9-30 (2001)

14. Chen, Z., Huan, G., Ma, Y.: Computational Methods for Multiphase Flows in Porous Media. SIAM, Philipadia (2006)

15. Ciarlet, P.G.: Mathematical Elasticity: Theory of Plates, vol. 2. Elsevier, Amsterdam (1997)

16. Formaggia, L., Fumagalli, A., Scotti, A., Ruffo, P.: A reduced model for Darcy's problem in networks of fractures. ESAIM: Math Model Numer Anal 48(4), 1089-1116 (2014)

17. Helmig, R.: Multiphase Flow and Transport Processes in the Subsurface: A Contribution to the Modeling of Hydrosystems. Springer, Berlin (1997)

18. Hiptmair, R.: Finite elements in computational electromagnetism. Acta Numerica 11, 237-339 (2002)

19. Jost, J.: Riemannian Geometry and Geometric Analysis. Springer, Heidelberg (1995)

20. LeVeque, R.J.: Numerical Methods for Conservation Laws. Birkhäuser, Boston (1992) 
21. Licht, M.W.: Complexes of discrete distributional differential forms and their homology theory. In: Foundations of Computational Mathematics (2016)

22. Lindell, I.V.: Differential Forms in Electromagnetics. IEEE Press, Piscataway (2004)

23. Martin, V., Jaffré, J., Roberts, J.E.: Modeling fractures and barriers as interfaces for flow in porous media. SIAM J Sci Comput 26(5), 1667-1691 (2005)

24. Melrose, R.B.: A remark on distributions and the de Rham theorem (2011). arXiv preprint arXiv :1105.2597

25. Mitrea, D., Mitrea, M., Shaw, M.C.: Traces of differential forms on Lipschitz domains, the boundary De Rham complex, and Hodge decompositions. Indiana Univ. Math. J. 57(5), 2061-2095 (2008)

26. Nordbotten, J.M.: Convergence of a cell-centered finite volume discretization for linear elasticity. SIAM J. Numer. Anal. 53(6), 2605-2625 (2016)

27. Nordbotten, J.M., Boon, W.M.: Modeling, structure and discretization of mixed-dimensional partial differential equations. In: Domain Decomposition Methods in Science and Engineering XXIV, Lecture Notes in Computational Science and Engineering (2017)

28. Nordbotten, J.M., Celia, M.A.: Geological Storage of $\mathrm{CO}_{2}$ : Modeling Approaches for Large-scale Simulation. Wiley, New York (2011)

29. Picard, R.: An elementary proof for a compact imbedding result in generalized electromagnetic theory. Mathematische Z. 187, 151-164 (1984)

30. Rudin, W.: Functional Analysis. International Series in Pure and Applied Mathematics. McGraw-Hill, New York (2006)

31. Russell, T.F., Wheeler, M.F.: Finite element and finite difference methods for continuous flows in porous media. In: Ewing, R.E. (ed.) Mathematics of Reservoir Simulation, pp. 35-106. SIAM, Philipadia (1983)

32. Spivak, M.: Calculus on Manifolds. Addison-Wesley, Reading (1965)

33. Toselli, A., Widlund, O.: Domain Decomposition Methods: Algorithms and Theory. No. 34 in Springer Series in Computational Mathematics, Springer (2005)

34. Weil, A.: Sur les théorèmes de de Rham. Commentarii Mathematici Helvetici 26, 119-145 (1952)

Publisher's Note Springer Nature remains neutral with regard to jurisdictional claims in published maps and institutional affiliations. 